\title{
THE EXTENDED HIGH $A(V)$ QUASAR SURVEY: SEARCHING FOR DUSTY ABSORBERS TOWARD MID-INFRARED-SELECTED QUASARS
}

\author{
J.-K. Krogager ${ }^{1,2}$, J. P. U. Fynbo ${ }^{2}$, K. E. Heintz ${ }^{2}$, S. Geier ${ }^{3}$, C. Ledoux ${ }^{4}$, P. Møller ${ }^{5}$, P. Noterdaeme ${ }^{1}$, \\ B. P. Venemans ${ }^{6}$, and M. VestergaARd ${ }^{2,7}$ \\ ${ }^{1}$ Institut d'Astrophysique de Paris, CNRS-UPMC, UMR7095, 98bis bd Arago, F-75014 Paris, France \\ ${ }^{2}$ Dark Cosmology Centre, Niels Bohr Institute, University of Copenhagen, Juliane Maries Vej 30, DK-2100 Copenhagen $\emptyset$, Denmark \\ ${ }^{3}$ Instituto de Astrofísica de Canarias (IAC), E-38205 La Laguna, Tenerife, Spain \\ ${ }^{4}$ European Southern Observatory, Alonso de Córdova 3107, Vitacura, Casilla 19001, Santiago 19, Chile \\ ${ }^{5}$ European Southern Observatory, Karl-Schwarzschildstrasse 2, D-85748 Garching bei München, Germany \\ ${ }^{6}$ Max-Planck Institute for Astronomy, Königstuhl 17, D-69117 Heidelberg, Germany \\ ${ }^{7}$ Steward Observatory and Department of Astronomy, University of Arizona, 933 N Cherry Avenue, Tucson, AZ 85721, USA \\ Received 2016 July 26; revised 2016 August 24; accepted 2016 August 29; published 2016 November 15
}

\begin{abstract}
We present the results of a new spectroscopic survey for dusty intervening absorption systems, particularly damped Ly $\alpha$ absorbers (DLAs), toward reddened quasars. The candidate quasars are selected from mid-infrared photometry from the Wide-field Infrared Survey Explorer combined with optical and near-infrared photometry. Out of 1073 candidates, we secure low-resolution spectra for 108 using the Nordic Optical Telescope on La Palma, Spain. Based on the spectra, we are able to classify 100 of the 108 targets as quasars. A large fraction (50\%) is observed to have broad absorption lines (BALs). Moreover, we find six quasars with strange breaks in their spectra, which are not consistent with regular dust reddening. Using template fitting, we infer the amount of reddening along each line of sight ranging from $A(V) \approx 0.1$ to $1.2 \mathrm{mag}$ (assuming a Small Magellanic Cloud extinction curve). In four cases, the reddening is consistent with dust exhibiting the $2175 \AA$ feature caused by an intervening absorber, and for two of these, an Mg II absorption system is observed at the best-fit absorption redshift. In the rest of the cases, the reddening is most likely intrinsic to the quasar. We observe no evidence for dusty DLAs in this survey. However, the large fraction of BAL quasars hampers the detection of absorption systems. Out of the 50 non-BAL quasars, only 28 have sufficiently high redshift to detect $\operatorname{Ly} \alpha$ in absorption.
\end{abstract}

Key words: galaxies: active - quasars: absorption lines - quasars: general

Supporting material: figure set

\section{INTRODUCTION}

The study of dust-reddened quasars, in terms of their number density and amount of dust extinction, provides important clues to the study of evolutionary scenarios for quasars and active galactic nuclei in general (e.g., Hopkins et al. 2005, and references therein). In most scenarios quasars are thought to start out as heavily dust-obscured phenomena before gradually clearing out the dust (by strong outflows and/or dust sublimation), leaving behind a population of unobscured, "typical" quasars (typical in the sense that they make up the bulk of known quasars; e.g., Sanders et al. 1988a, 1988b; Hopkins et al. 2006).

In addition to the intrinsic variations in the quasar environments, any intervening dust in galaxies along the line of sight (including our own Milky Way) causes the background quasar to appear dust reddened as well (Fall \& Pei 1989; Fall et al. 1989; Pei et al. 1991). Such intervening galaxies are typically associated with the so-called damped Ly $\alpha$ absorbers (DLAs; see Wolfe et al. 2005). By studying the absorption in the spectrum of the background quasar, we can recover important quantities about the absorbing gas, e.g., its metallicity, ionization state, dust depletion, and in some cases even the density and temperature (e.g., Howk et al. 2005; Srianand et al. 2005; Jorgenson et al. 2010; Kanekar et al. 2014; Cooke et al. 2015; Neeleman et al. 2015; Noterdaeme et al. 2015b). From looking at depletion in large samples of DLAs it has been shown that the more chemically evolved DLAs (i.e., with high metallicity) are more likely to harbor dust (Ledoux et al. 2003; De Cia et al. 2013). Thus, the metal-rich and dusty DLAs hold valuable information about the most chemically enriched systems and allow us to study in great detail the molecular absorption (since molecules are primarily formed on the surface of dust grains; Jenkins \& Peimbert 1997) and the cold gas phase of the absorbing gas (e.g., Srianand et al. 2005; Noterdaeme et al. 2007).

The vast majority of quasars (and thereby also DLAs; Prochaska et al. 2005; Prochaska \& Wolfe 2009; Noterdaeme et al. 2009, 2012b) have been identified in optical, large-area sky surveys, most notably the Sloan Digital Sky Survey (SDSS; York et al. 2000, as well as its extension, the Baryon Oscillation Spectroscopic Survey [BOSS]; Dawson et al. 2013) and the $2 \mathrm{dF}$ QSO redshift survey (Croom et al. 2004). The power of selecting quasars in the optical is proven by the sheer number of quasars identified in the SDSS catalog. This optical selection mainly relies on the fact that quasars have very blue optical colors. However, the technique falls short for high redshifts where stars and quasars are no longer easily discernible in the optical color space. Also, if the quasar has intrinsic spectral differences relative to the "typical" quasar population, e.g., dust reddening, weak emission lines, or broad absorption lines (BALs), the quasar will appear redder in the observed optical emission, making the distinction between stars and quasars even more difficult (Richards et al. 2002, 2003). Furthermore, a dust-rich absorption system along the line of sight can cause a significant reddening effect on the background quasar (e.g., Fall et al. 1989; Noterdaeme et al. 2009, 2012a; Jiang et al. 2010; Kaplan et al. 2010; Fynbo et al. 2011; Wang et al. 2012; Krogager et al. 2016). All of these effects 
may cause the background quasar to change appearance to the point where optical searches become blind to these reddened objects. This introduces a bias in the parent quasar sample from which DLAs are selected. As a result, this bias propagates to a bias in the DLA samples, since the quasars behind chemically evolved and dusty galaxies are missed more often than quasars behind dust-poor galaxies.

The mere existence of this bias and its potential impact on the DLA population have been studied in great detail in the literature (Fall \& Pei 1989; Fall et al. 1989; Pei et al. 1991; Ellison et al. 2001; Murphy \& Liske 2004; Vladilo \& Péroux 2005; Vladilo et al. 2008; Jorgenson et al. 2006; Frank \& Péroux 2010; Pontzen \& Pettini 2009; Kaplan et al. 2010; Khare et al. 2012; Murphy \& Bernet 2016). A large part of these studies have been based on optically selected samples, which are not sensitive to this hidden population of dusty DLAs. However, the surveys by Ellison et al. (2001) and Jorgenson et al. (2006) were carried out at radio wavelength since the radio emission from quasars is not affected by dust. While they find that dust obscuration is a small effect (for the incidence rate of DLAs), they also conclude that larger radio samples are needed to firmly conclude on the importance of a dust bias (see also the analyses of Ellison et al. 2005, 2008).

In order to gauge the population of reddened quasars, a substantial number of surveys have been undertaken using various selection methods (e.g., Benn et al. 1998; Warren et al. 2000; Gregg et al. 2002; Richards et al. 2003; Hopkins et al. 2004; Polletta et al. 2006; Glikman et al. 2007, 2012, 2013; Lacy et al. 2007; Maddox et al. 2008, 2012; Urrutia et al. 2009; Banerji et al. 2012; Hainline et al. 2014), and, more recently, wide-field, infrared facilities, such as the UKIRT Infrared Deep Sky Survey (UKIDSS; Lawrence et al. 2007), the VISTA Kilo-Degree Infrared Galaxy Survey (VIKING; Fleuren et al. 2012), and the Wide-field Infrared Survey Explorer (WISE; Wright et al. 2010), allow quasar candidates to be selected in great numbers based on the shape of their spectral energy distributions (SEDs) instead of using just one single characteristic (e.g., Warren et al. 2000, 2007; Peth et al. 2011; Maddox et al. 2012). While such efforts are very effective for photometric classification (Richards et al. 2015; Peters et al. 2015), the spectroscopic follow-up and classification are much more time-consuming. The BOSS program has provided a massive improvement in terms of a more unbiased quasar selection due to the development of complex multiwavelength and multi-epoch selection algorithms (Ross et al. 2012, and references therein), but the red quasar population is still quite sparsely sampled.

In contrast to most other surveys for red quasars, which in various ways focus on a complete census of the quasar population, we initiated our spectroscopic survey (High $A(V)$ Quasar [HAQ] Survey) with the primary focus on finding dustrich foreground absorption systems. In the first surveys, we used near-infrared (NIR) and optical photometry alone to select point sources that are consistent with "typical" quasars reddened by a foreground dusty absorber. The initial strategy is laid out by Fynbo et al. (2013, hereafter Paper I), and a refined set of those criteria were used for a much larger survey presented by Krogager et al. (2015, hereafter Paper II). In accordance with previous works analyzing the DLA dust bias, we find in the HAQ survey that the incidence rate of dusty absorbers is low, i.e., the dusty foreground absorbers do not lead to a strong bias in terms of the $N(\mathrm{HI})$ distribution; however, since these dusty DLAs are preferentially metal-rich (e.g., Fynbo et al. 2011; Krogager et al. 2016), the missing dusty DLAs are important for the overall metal distribution (see also Pontzen \& Pettini 2009; Khare et al. 2012). Furthermore, the dusty DLAs are important for a complete census of the cold and molecular gas phases in absorption-selected galaxies (Ledoux et al. 2015).

In this work, we present the extended HAQ (eHAQ) survey, designed to probe quasars with more reddening relative to Paper II (this part of color space was initially included in Paper I, but this led to a large fraction of stellar and galactic contamination) and to eliminate $z \lesssim 1.5$ quasars in order to be able to detect the Ly $\alpha$ absorption line in our observed spectra. The new selection criteria presented in the following allow us to select a purer sample of reddened quasar candidates by basing the sample selection on WISE mid-infrared (MIR) photometry. Furthermore, we are able to effectively remove low-redshift quasars as desired. In the following, we present the results of our spectroscopic follow-up campaign of 108 candidates from our new criteria.

Section 2 deals with the photometric data used in our work, our selection criteria, and our proposed method to remove stellar contamination. In Sections 3 and 4, we describe our spectroscopic observations, together with our classifications and analyses of the sample. In Section 5, we discuss the implications of our work. Throughout this work, we will assume a flat $\Lambda \mathrm{CDM}$ cosmology with $H_{0}=67.9 \mathrm{~km} \mathrm{~s}^{-1} \mathrm{Mpc}^{-1}, \Omega_{\Lambda}=0.69$, and $\Omega_{\mathrm{M}}=0.31$ (Planck Collaboration 2014).

\section{PHOTOMETRIC DATA AND TARGET SELECTION}

The photometric catalog was compiled by cross-matching the SDSS Data Release $8(u, g, r, i$, and $z$ bands at $0.36,0.47,0.62$, 0.75 , and $0.90 \mu \mathrm{m}$, respectively), the UKIDSS Data Release 10 $\left(Y, J, H\right.$, and $K_{s}$ bands at $1.03,1.25,1.64$, and $2.21 \mu \mathrm{m}$, respectively), and the WISE AllSKY data release $\left(W_{1}, W_{2}, W_{3}\right.$, and $W_{4}$ at 3.4, 4.6, 12, and $22 \mu \mathrm{m}$, respectively). In the following, we will refer to the $K_{s}$ band of UKIDSS simply as the $K$ band.

We matched this optical/infrared catalog to the Faint Images of the Radio Sky at Twenty cm (FIRST, data release 14Dec17) to compare radio properties of our parent sample and the SDSS/BOSS targets. We stress that the radio data were only included as ancillary data and were not part of the sample selection criteria. The survey provides radio coverage at $1.4 \mathrm{GHz}$ with an average sensitivity of $\sim 0.15 \mathrm{mJy}$ ( $\mathrm{rms}$ ).

The selection of candidate quasars was based primarily on MIR photometry from WISE. We required the candidates to be detected with a signal-to-noise ratio of at least 5 in the first three WISE bands (i.e., $\mathrm{S} / \mathrm{R}_{W 1-3} \geqslant 5$ ). Moreover, we require that the candidates are detected (at more than $3 \sigma$ significance) in $g, r, i, J$, and $K$, and that they are point sources in both UKIDSS and SDSS (specifically, we use the catalog flags: mergedclass $=-1$ and sdsstype $=6$ ). We limited our follow-up to candidates that had not already been observed spectroscopically either by us in Papers I and II or in SDSS or BOSS. The declination of the candidates was restricted to be in the range from $-4^{\circ}$ to $+17^{\circ}$ in order to allow follow-up from facilities in the Southern Hemisphere while still being accessible to the Nordic Optical Telescope (NOT).

Based on experience from the previous surveys (Paper II), we have created a selection cut in the $W_{1}-W_{2}$ versus $W_{2}-W_{3}$ color-color space in order to select candidates with redshifts larger than $z>1.5$. From the distribution of redshifts of the quasars identified in Paper II, we were able to define a cut in the 


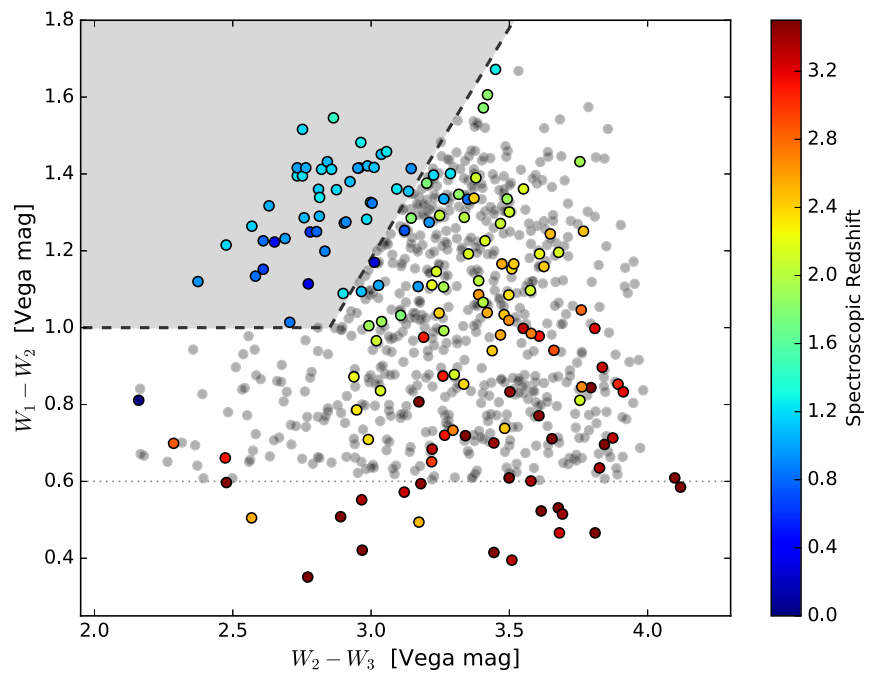

Figure 1. WISE color-color plots for this work and Paper II. Each colored point corresponds to a quasar from HAQ (Paper II), and its color indicates the spectroscopic redshift. Gray points are candidates for this work. The gray region in the upper left corner indicates the exclusion zone for low-redshift quasars. The dotted line marks the lower limit on $W_{1}-W_{2}$ color in order to limit contamination from stars and galaxies.

WISE color space to exclude low-redshift quasars; see Figure 1. We imposed the following color cuts in WISE:

for $W_{2}-W_{3}<2.85: W_{1}-W_{2}<1$,

for $W_{2}-W_{3} \geqslant 2.85: \quad W_{1}-W_{2}<1.2 \times\left(W_{2}-W_{3}\right)-2.42$,

$W_{1}-W_{2}>0.6$ and $2<W_{2}-W_{3}<4$.

The lower boundary on the $W_{1}-W_{2}$ color was imposed to limit contamination from stars and galaxies while still recovering a part of the high-redshift quasars. This was motivated by the results of Stern et al. (2012); see also discussion by Richards et al. (2015).

Since we wish to target quasar candidates with potential dusty foreground absorbers, we include two optical color cuts to ensure that the candidates are optically red (consistent with dust reddening): $g-r>0.5$ and $r-i>0.4$ (i.e., the same as in Papers I and II).

In the first observing run, we limited our selection to $J_{\mathrm{AB}}<19$. In the subsequent runs we relaxed this magnitude cut; however, only a small number of observed candidates have $J_{\mathrm{AB}}>19$ (5 out of 108 observed).

\subsection{Stellar Rejection}

During the first observing run (P50-802), we identified a significant fraction of contamination from stars. The majority of these contaminants were observed to have $J-K<0$. We therefore imposed an additional NIR color cut $(J-K>-0.05)$ for the subsequent spectroscopic observations in order to remove stellar contaminants. This is illustrated in Figure 2. All targets selected after P50-802 have been corrected for the stellar contaminants using the NIR cut.

\section{SPECTROSCOPIC OBSERVATIONS}

During a series of observing runs in 2015 and 2016, a total of 108 candidate quasars were observed with the $\mathrm{NOT}^{8}$ using the Andalucía Faint Object Spectrograph and Camera

\footnotetext{
8 Located at the Observatorio del Roque de los Muchachos on La Palma, Spain.
}

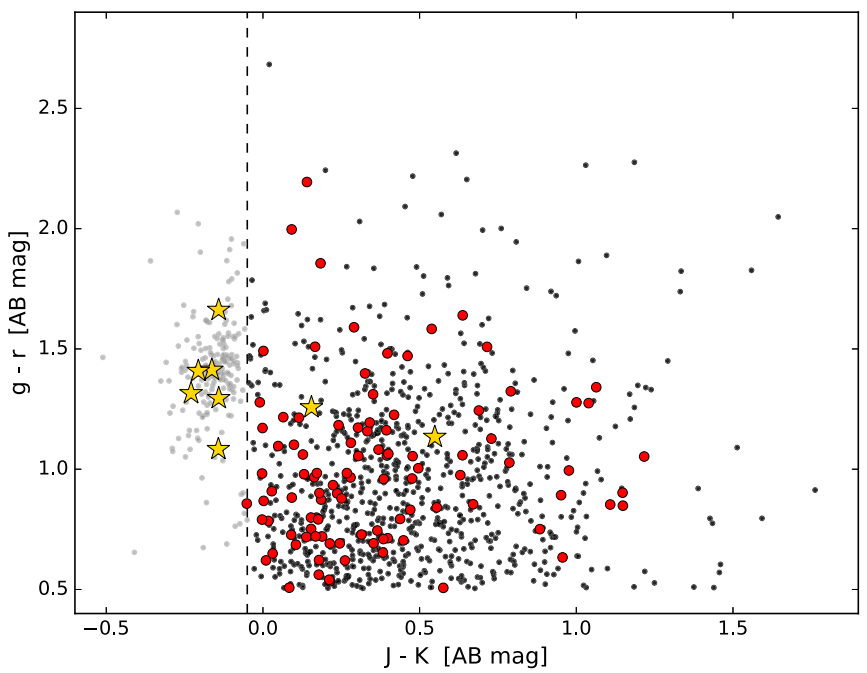

Figure 2. Color-color plot showing optical $g-r$ color vs. NIR $J-K$ color for the eHAQ candidates. The small points show the full parent sample, where quasars and stars (based on photometry) are shown by black and gray points, respectively. The imposed photometric criterion $(J-K>-0.05)$ to reject stars is shown by the vertical dashed line. Large points indicate the targets that were spectroscopically observed: quasars (red circles) and stars (yellow stars).

(ALFOSC). For all the ALFOSC observations, we binned the CCD pixels by a factor of 2 along the wavelength axis and aligned the slit with the parallactic angle. The candidates were, as in Paper II, observed using grism \#4, which covers the wavelength range from about 3200 to $9100 \AA$ at a resolution of about $R \sim 300$ with a slit width of 1 ."3. Redward of about $7500 \AA$ the spectra are strongly affected by fringing, which was alleviated by dithering along the slit. In order to prevent second-order contamination from wavelengths shorter than $3500 \AA$, a blocking filter was used for the observations with grism \#4. We used filter no. 94, which blocks out wavelengths shorter than $3560 \AA$. Given the red nature of the targets (by selection) and the reduced intensity of the second-order spectrum, the contamination from the remaining flux that is not blocked by the filter (from 3560 to $4500 \AA$ ) will be low (however, it may still be an issue, as observed in Heintz et al. 2016). The overlap from the second order starts at $7120 \AA$, which is furthermore the spectral range affected by strong fringing. Moreover, we use the overall spectral shape from the broadband photometry to make sure that we are not affected by significant second-order contamination. Based on this consistency check, we find the second-order contamination to be negligible. One candidate (eHAQ2359+1354) was observed with grism \#6 alone to investigate tentative signs of a DLA seen in the spectrum obtained by BOSS. Grism \#6 covers wavelengths from 3200 to $5810 \AA$ at a resolution of about $R \sim 500$ with the 1 ". 0 slit.

Four additional candidates were observed with the Intermediate Dispersion Spectrograph (IDS) situated at the Cassegrain focus of the Isaac Newton Telescope (INT). For spectra obtained with the IDS, we used the grating "R400R," which covers wavelengths from 5000 to $9500 \AA$ at a resolution of roughly $R \sim 2250$.

Details about the spectroscopic observations are provided in Table 3 in Appendix A. In the table, we also indicate overlap with existing spectroscopy from BOSS. The overlap was caused by a mistake in the cross-referencing for the target selection. 


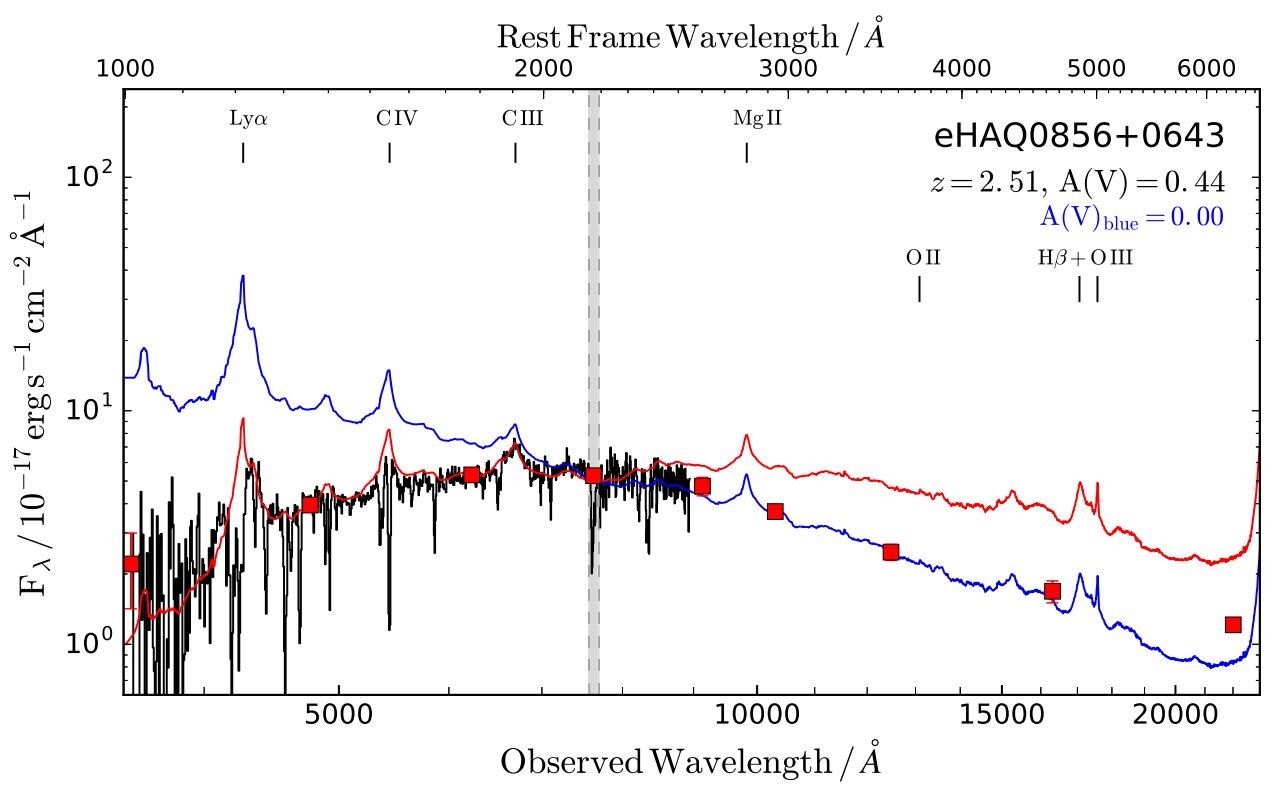

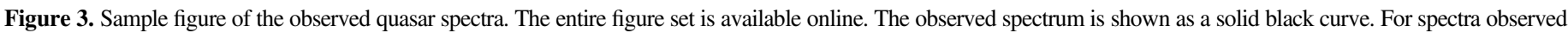

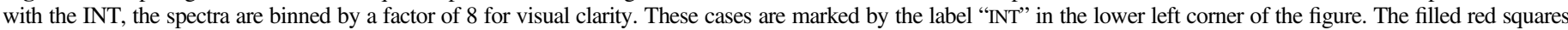

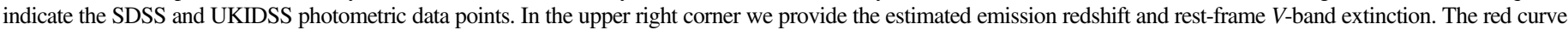

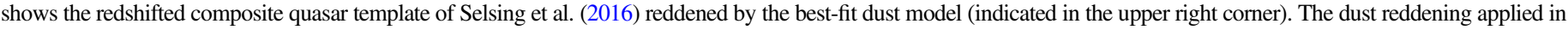

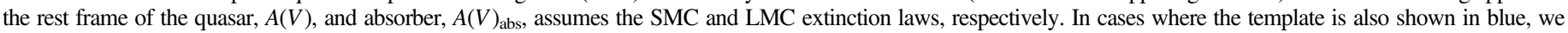

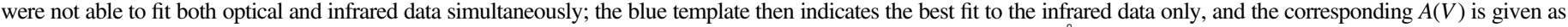
$A(V)_{\text {blue }}$. Note that the spectra have not been corrected for telluric absorption (marked with a gray band at $\sim 7600 \AA$ ).

(The complete figure set (100 images) is available.)

\subsection{Data Reduction}

The spectra were reduced using a combination of $\operatorname{IRAF}^{9}$ and MIDAS $^{10}$ tasks for low-resolution spectroscopy. Cosmic rays were rejected using the software written by van Dokkum (2001). In case of photometric observing conditions the spectrophotometric standard star observed on the same night as the science spectra was used for the flux calibration. Otherwise, we used a standard response curve to calibrate the spectra. The spectra and photometry were corrected for Galactic extinction using the extinction maps from Schlafly \& Finkbeiner (2011). In order to improve the absolute flux calibration, we scaled the spectra to the $r$-band photometry from SDSS.

For the four targets observed with the INT, we were not able to recover a robust flux calibration. The shape of the spectra does not match the observed photometry from SDSS. However, the wavelength calibration is robust. We therefore only use the spectra to measure the spectroscopic redshifts.

\section{RESULTS}

For all but two candidates, we were able to securely classify the object as either a quasar or star based on the available data. We identify eight stars (one of which is tentative) and 100 quasars (one of which is tentative). Individual notes for each target are given in Appendix D. Below we describe the stellar and quasar spectra in more detail.

\footnotetext{
9 IRAF is distributed by the National Optical Astronomy Observatory, which is operated by the Association of Universities for Research in Astronomy (AURA) under cooperative agreement with the National Science Foundation.

${ }^{10}$ ESO-MIDAS is a copyright-protected software product of the European Southern Observatory. The software is available under the GNU General Public License.
}

\subsection{Quasars}

The spectra of the 100 quasars are shown in Figure 3, together with the SDSS and UKIDSS photometry. For every quasar we measure the redshift from the characteristic broad emission lines by template matching. In a few cases where the broad emission lines are heavily suppressed (due to BAL or intrinsically weak emission lines), we use absorption features to estimate the quasar's systemic redshift. In order to infer the reddening of each quasar, we fit the spectrum with the quasar template from Selsing et al. (2016) following the same approach as described in Paper II assuming the extinction law for the Small Magellanic Cloud (SMC; $R_{V}=2.74$ ) by Gordon et al. (2003). We use the SMC extinction curve since we see no evidence for the $2175 \AA$ dust bump in the observed spectra. Moreover, we note that using the steeper extinction curve derived by Zafar et al. (2015) leads to consistent fits only with a lower best-fit value of $A(V)$ due to the lower value of $R_{V}=2.41$ for this extinction curve; in terms of $E(B-V)$ the best-fit values are identical. Since the two curves only differ significantly blueward of C III, it is only possible to distinguish the two curves in spectra of quasars at redshifts larger than $z \gtrsim 1.8$. However, here the intrinsic variations in emission lines (and BAL features) prevent us from significantly distinguishing one from the other due to the limited resolution and signal-to-noise ratio of our data. One exception is the photometric fits performed in Section 4.1.3 since for these quasars the weak emission lines do not cause large variations in the SED. Only in a few cases do we see evidence for a steeper extinction curve and apply the extinction curve from Zafar et al. (marked with b in Table 4 in Appendix A). For consistency with other works and with Papers I and II, we use the SMC extinction curve in this work, unless stated otherwise. As noted in Papers I and II (as well as other works; e.g., Urrutia et al. 2009), the $A(V)$ values derived from template fitting are mostly indicative since the main source of uncertainty comes from the assumption 
that all quasars follow the same template. We find an average statistical uncertainty of $0.01-0.02 \mathrm{mag}$ for the signal-to-noise ratio of our data (see also Section 4.1.2 in Paper II). By varying the intrinsic slope of the quasar template before fitting, we recover an estimate of the systematic uncertainty due to these intrinsic variations of around $0.02 \mathrm{mag}$. Moreover, the best-fit $A(V)$ is correlated with the assumed value of $R_{V}$ for the SMC or Zafar et al. (2015) curves. For the Zafar et al. (2015) extinction curve $\left(R_{V}=\right.$ 2.41 ), the corresponding extinction, $A(V)_{\mathrm{Z} 15}$, is related to the bestfit extinction given in this work, $A(V)_{\mathrm{SMC}}$, by the following expression:

$$
A(V)_{\mathrm{Z} 15}=\frac{R_{V_{\mathrm{Z} 15}}}{R_{V_{\mathrm{SMC}}}} A(V)_{\mathrm{SMC}}=0.88 A(V)_{\mathrm{SMC}} .
$$

The inferred quasar redshift and $A(V)$ are given in the upper right corner of Figure 3. For the target eHAQ2359+1354, we use the available BOSS spectrum to fit the extinction, since the grism \#6 spectrum does not allow us to constrain the reddening. In some cases, we are not able to fit both the optical spectrum and the NIR photometry simultaneously, and we therefore provide an $A(V)$ value for both the optical and NIR fits. These peculiar cases are presented later in more detail. The spectroscopic redshift, the best-fit $A(V)$ (assuming SMC-type dust), and our spectral classification are given in Table 4 in Appendix A. We caution that the BAL classification does not follow a rigorous scheme; it is merely judged by eye in the spectra.

Figure 4 shows the distribution of redshifts and visual extinction, $A(V)$, for the current quasar sample. For comparison, we also show the sample from Paper II. It is clear that the inclusion of WISE photometry has enabled us to probe higher amounts of reddening and reject low-redshift quasars while still having a very low level of stellar contamination in our candidate sample.

\subsubsection{Intervening Dust}

Since our main focus for this survey is the intervening dusty systems, we search for absorption systems in the spectra. Table 1 lists the spectroscopic absorption redshift for all the identified absorption systems in this sample. As in Paper II, we model each spectrum using a combined dust model with a freely variable dusty absorber and an amount of extinction fixed in the quasar rest frame. The details of this fitting procedure are described in Paper II. Four quasars are selected by our statistical algorithm as having an intervening absorption system hosting LMC-type dust; see individual fits in Appendix C. These four statistically identified absorption systems are given in Table 1 , together with the spectroscopically identified intervening absorption systems. Two of the statistically identified systems match the spectroscopically identified redshift well within the $1 \sigma$ uncertainty of the fit. The other two show no absorption at the best-fit redshift. We identify no absorbing systems with SMC-type reddening, which is to be expected due to the degeneracy between dust in the quasar itself (assumed to be SMC type) and dust in the absorber (see Paper II). Two plausible DLAs are identified (eHAQ0930+0148 and eHAQ2359+1354) with intermediate amounts of SMC-type dust reddening along the line of sight. However, we observe no evidence for the dust reddening being caused by the absorber. Due to the poor spectral resolution, it is not possible to securely obtain the column densities of $\mathrm{H}$ I from $\operatorname{Ly} \alpha$.

\subsubsection{Peculiar Quasars}

As mentioned above, some quasars are not well described by the quasar template assuming SMC-type reddening. The optical

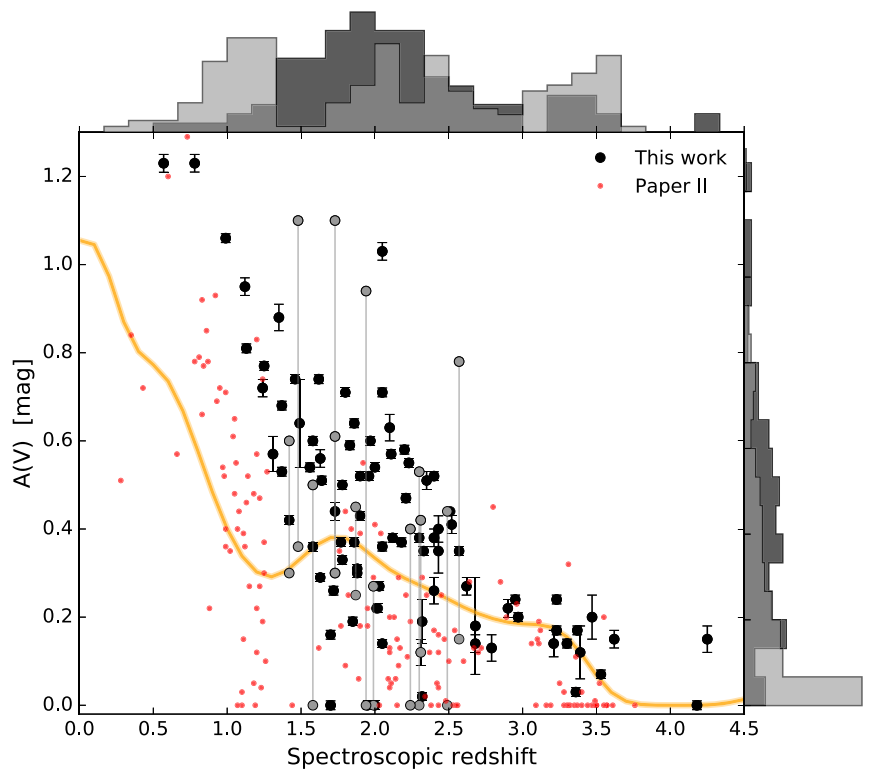

Figure 4. Distribution of redshift vs. the amount of $V$-band extinction, $A(V)$, from the spectral fitting. The large filled circles show the sample from this work. The error bar (typically $0.01 \mathrm{mag}$ ) is smaller than the point in most cases. The small red points are taken from Paper II. The points that are connected with a gray line refer to the peculiar quasars in Table 4 for which a range of $A(V)$ is given; see Section 4.1 for details. For clarity, the corresponding points are shown in gray. The orange curve shows the value of $A(V)$ assuming SMC-type extinction for which a quasar at the given redshift has an observed color of $g-r=0.5 \mathrm{mag}$. Since one of our criteria is $g-r>0.5$, points below the curve will therefore enter our sample due to a mechanism other than pure dust reddening, e.g., strong BAL features or weak emission lines. The histograms on the top show the redshift probability density functions (PDFs) of this work (dark gray) and of Paper II (lightgray overlapping). Similarly, the histograms on the right show the PDFs of $A(V)$.

spectrum, in most cases, requires a higher amount of $A(V)$ than what is needed to fit the NIR photometry. This is indicated in Table 4 in Appendix A as a range in $A(V)$. We have tried to apply the steeper extinction curve inferred by Zafar et al. (2015), but this does not provide a good fit either (except for two cases, marked by $b$ in Table 4 in Appendix A). The extinction curve needed to fit these peculiar objects is even steeper than the curve by Zafar et al. and probably exhibits a break in order to reconcile the data.

For two of these peculiar quasars (where we do not observe strong absorption), we attempt to recover the reddening curve in order to study the sightlines in more detail; see Figure 5. We stress that since we do not know the absolute intrinsic normalization for these lines of sight, we only infer the relative reddening curve and not the absolute extinction.

First, we normalize the quasar template (Selsing et al. 2016) to the $K$ band, and then we calculate the relative reddening, $k_{\lambda}$, as

$$
k_{\lambda}=-2.5 \log \left(D_{\lambda} / T_{\lambda}\right)
$$

where $D_{\lambda}$ refers to the observed data and $T_{\lambda}$ refers to the flux predicted from the template. For photometric data, we convolve the template with the corresponding filter transmission curves to obtain synthetic photometry. The resulting curves are shown in Figure 5, where we compare with the reddening curves of SMC-type dust (Gordon et al. 2003) and quasar-type dust (Zafar et al. 2015), both arbitrarily normalized to illustrate the differences in slope. It is clear that the two known reddening laws provide an equally inadequate description of the data. Both curves can be made to fit part of the data, by correctly normalizing the reddening curve; however, this can only match 

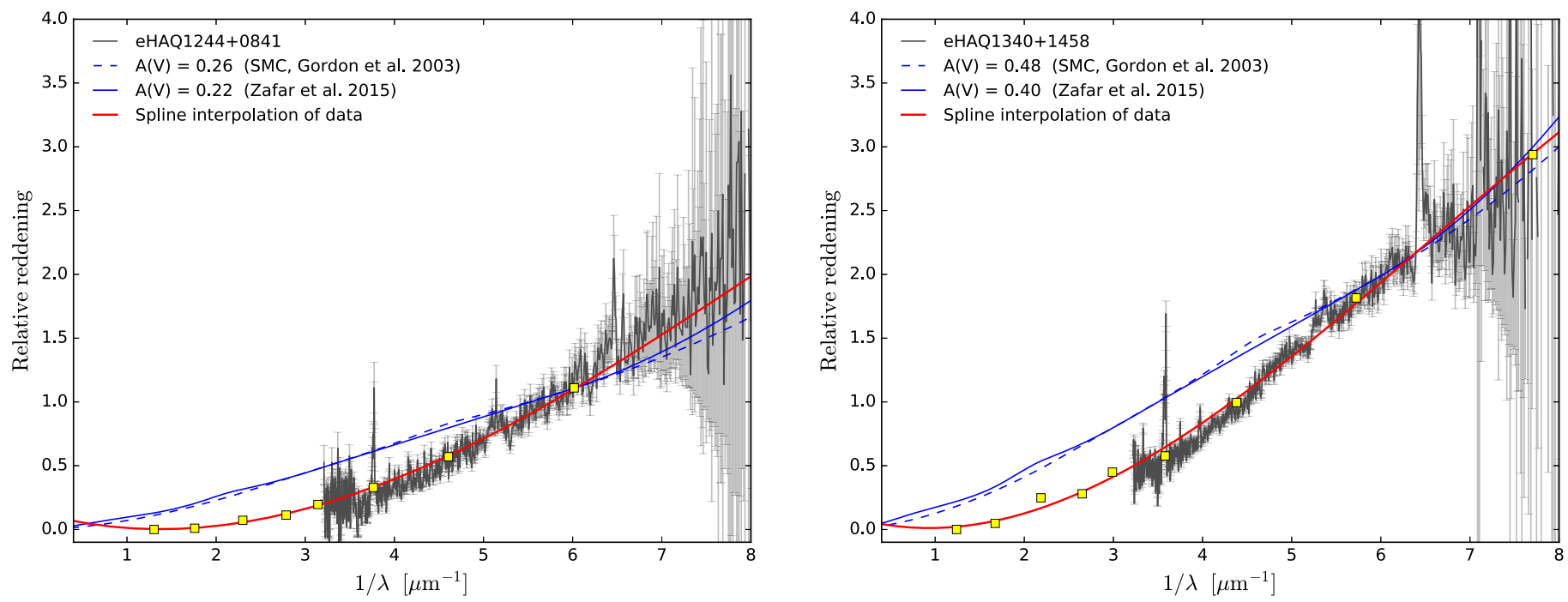

Figure 5. Reddening curves for two quasars, eHAQ1244+0841 (left) and eHAQ1340+1458 (right), shown in the rest frame of the quasars. The spectral data are shown as gray lines with error bars, and the broadband photometry is shown as yellow squares. Since we do not know the intrinsic brightness of the quasar, the data have been normalized, arbitrarily, to the $K$ band. The data thus only indicate the relative reddening and not the total extinction. The blue curves show two dust models with varying amounts of reddening to best match the data; however, it is clear that they do not match the curvature of the data. For comparison, we highlight the shape of the reddening curve inferred from the broadband photometry by a spline interpolation (shown in red). The prominent curvature around $\lambda^{-1}=3.5 \mu \mathrm{m}^{-1}$ gives rise to the so-called $3000 \AA$ break in the observed spectra reddened by this type of extinction.

Table 1

Intervening Absorption Systems

\begin{tabular}{lccccc}
\hline \hline Target & $z_{\text {spec }}{ }^{\mathrm{a}}$ & $z_{\text {abs }}{ }^{\mathrm{b}}$ & $A(V)_{\text {abs }}{ }^{\mathrm{b}}$ & $A(V)_{\mathrm{QSO}}$ & Notes \\
\hline eHAQ0019+0657 & 1.671 & $\ldots$ & $\ldots$ & 0.17 & Mg II absorber \\
eHAQ0104+0912 & 1.487 & $1.52 \pm 0.03$ & 0.20 & 0.16 & Mg II absorber \\
eHAQ0111+0641 & 2.027 & $2.04 \pm 0.09$ & 0.21 & 0.15 & Fe II absorption \\
eHAQ0113+0804 & 1.589 & $\ldots$ & $\ldots$ & 0.60 & Mg II absorber \\
eHAQ0321+0523 & $\ldots$ & $\ldots .02 \pm 0.02$ & $\ldots$ & 0.15 & No absorption lines in spectrum \\
eHAQ0835+0127 & 1.717 & $\ldots$ & $\ldots$ & 0.14 & Fe II absorption, tentative \\
eHAQ0930+0148 & 2.720 & $\ldots$ & $\ldots$ & 0.24 & Si II absorption, Ly $\alpha$ \\
eHAQ1109+1058 & 1.667 & $1.22 \pm 0.01$ & $\ldots$ & 0.43 & Mg II absorber \\
eHAQ1455+0705 & 1.650 & $\ldots$ & 0.23 & Mg II absorber \\
eHAQ2359+1354 & $2.248^{\mathrm{c}}$ & & $\ldots .13$ & Mg II absorber, Ly $\alpha$ \\
\hline
\end{tabular}

Notes.

${ }^{\text {a }}$ Spectroscopically identified absorption redshift.

${ }^{\mathrm{b}}$ Best-fit parameters from intervening dust model. Only cases for which dust in an intervening absorber was preferred are shown. $A(V)_{\text {abs }}$ assumes LMC-type dust in the rest frame of the absorber.

${ }^{c}$ Based on the spectrum from BOSS.

either the infrared or the optical data — not both simultaneously. The extinction curve needed to match the observed, peculiar spectra therefore needs a different curvature relative to the SMC-type curve or the curve by Zafar et al. (2015).

We also identify some targets with very strong BAL features, which makes it impossible to determine the intrinsic continuum (e.g., eHAQ0104+0756, eHAQ0044+1321, eHAQ0913+0910). Similarly, we identify a few weak-line quasars for which the quasar template provides a bad description since the template was compiled from luminous quasars with regular emission lines. The reddening estimates in these cases are therefore not representative.

Another type of object denoted as peculiar are the quasars for which the template seems to provide a bad match over a small spectral range. This is typically caused by intrinsic variations in the pseudo-continuum caused by a large blend of broad iron emission lines from Fe II and Fe III and can to some degree be accounted for by including the emission template from Vestergaard \& Wilkes (2001).
For all these peculiar cases we mark the quasar by an a in Table 4 in Appendix A. The reddening measurement should in these cases be considered rough estimates, which will need further investigation over a larger wavelength baseline. For individual comments about the quasars, we refer the reader to Appendix D.

\subsubsection{Photometric Fitting of Weak Emission Line Quasars}

For two quasars (eHAQ0839+0556 and eHAQ1340+0151) classified as peculiar, we fit the photometry alone, disregarding the spectrum, because the emission lines are very weak and barely visible. Since the spectrum exhibits no emission features, we use the continuum template of Richards et al. (2006) and apply reddening using the extinction curve of Zafar et al. (2015), as the slightly different curvature of this extinction curve compared to the SMC curve provides a better fit to the rest-frame UV data. Since both of these quasars appear to have little reddening in the NIR photometry but a high amount of reddening in the optical spectrum, we fit the spectra 

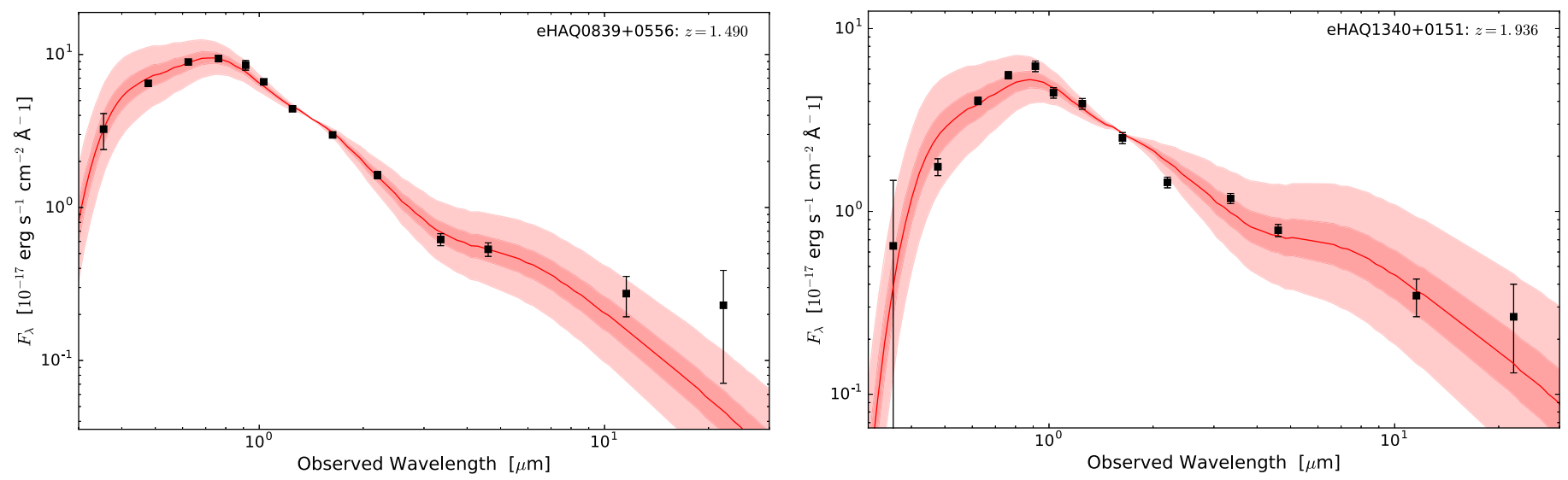

Figure 6. SED of the weak-line quasar eHAQ0839+0556 (left) and the target, eHAQ1340+0151, tentatively identified as a weak-line quasar (right). Broadband photometric data are shown as black squares with error bars. The best-fit model is shown by the red curve surrounded by the $1 \sigma$ and $3 \sigma$ confidence intervals shown by dark- and light-red shaded areas, respectively.

Table 2

SED Modeling of Weak-line Quasars

\begin{tabular}{lcccrr}
\hline \hline Target & $z$ & $\Delta \beta^{\mathrm{a}}$ & $A(V)$ & $R_{V}$ & $\log (s)^{\mathrm{b}}$ \\
\hline eHAQ0839+0556 & 1.49 & $-0.88 \pm 0.09$ & $0.64 \pm 0.10$ & $2.04 \pm 0.25$ & $-0.75 \pm 0.04$ \\
eHAQ1340+0151 & $1.82 \pm 0.11$ & $-0.49 \pm 0.11$ & $0.85 \pm 0.11$ & $2.66 \pm 0.28$ & $-0.82 \pm 0.05$ \\
\hline
\end{tabular}

Notes.

${ }^{\mathrm{a}}$ Offset in power-law index relative to the intrinsic quasar template. For $\Delta \beta<0$, the resulting template spectrum is steeper.

${ }^{\mathrm{b}}$ The nuisance parameter, $s$, is an arbitrary scale factor in order to match the template to the absolute flux scale of the data.

allowing the intrinsic power-law slope of the quasar and the steepness $\left(R_{V}\right)$ of the extinction curve to vary. We parameterize the change in the power-law slope by the offset, $\Delta \beta$, relative to the intrinsic power-law slope of the template, $\alpha_{\lambda}=-1.7$ (measured from the template by Richards et al. in the rest-frame wavelength range from 0.1 to $1.0 \mu \mathrm{m}$ ), where $f_{\lambda} \propto \lambda^{\alpha_{\lambda}}$.

Since all these parameters are highly degenerate, we use a Markov chain Monte Carlo routine ${ }^{11}$ to constrain the parameters using realistic priors for the power-law slope and $R_{V}$. For the quasar power-law slope, we use a Gaussian prior centered around $\Delta \beta=0$ with a $1 \sigma$ width of 0.2 (Krawczyk et al. 2015; Selsing et al. 2016). Similarly, for $R_{V}$ we use a Gaussian prior centered on $R_{V}=2.4$ with a $1 \sigma$ width of 0.3 (Zafar et al. 2015). Since the spectral identification for eHAQ1340+0151 is merely tentative, we allow the redshift to vary. The resulting best fits are shown in Figure 6. In both cases, the model performs very well and demonstrates that these weak-line quasars can be described by a continuum-only model. The best-fit parameters for both quasars are listed in Table 2 . The best-fit relative slopes $(\Delta \beta)$ both result in a much steeper intrinsic quasar template needed to fit the data; however, these values of $\Delta \beta$ are heavily degenerate with the shape parameter of the extinction curve, $R_{V}$, and should be considered indicative only. We also caution the reader that while the continuum-only model seems to provide a good description of the data, the template by Richards et al. (2006) was compiled from a sample of regular type 1 quasars and hence might not capture the nature of this type of weak-line quasar adequately.

\subsection{Stellar Contaminants}

The stellar spectra are easily recognizable, except for a few cases (see below). We classify the stellar spectra using an

\footnotetext{
${ }^{11}$ We use the Python code emcee written by Foreman-Mackey et al. (2013)
}

automated algorithm that fits a library of stellar templates (Pickles 1998) to the spectra. The best-fit spectral type for each target is given in Appendix B. The individual stellar spectra and the broadband photometry are also shown in Appendix B.

For one target ("eHAQ1132+1243") the classification is rather uncertain, due to the lack of strong spectral features. The target is classified as a metal-rich K4 giant. An alternative explanation could be that the target is a quasar at redshift $z=2.26$ with no strong emission lines. This is roughly consistent with the photometric redshift from quasar template fitting: $z_{\text {phot }}=3.6 \pm$ 0.9. Moreover, the quasar template from Richards et al. (2006) provides a better fit to the WISE photometry, and the quasar nature would more easily explain the apparent jump between the SDSS and UKIDSS photometry as intrinsic variability between the different epochs of observation in the two surveys. However, more data are needed to securely identify this target.

As mentioned in Section 2, we note that all the stellar contaminants have $J-K<0$, except for the two targets classified as $\mathrm{K}$ giants, including the just-mentioned insecure identification.

\section{DISCUSSION}

In this work, we present the results of a new spectroscopic survey, the eHAQ survey, targeting reddened quasars with the aim of finding dust-rich absorption systems. By selecting point sources from a cross-matched catalog of SDSS, UKIDSS, and WISE, we have compiled a sample of 1073 quasar candidates based on their MIR properties. Motivated by results from the previous HAQ survey (Paper II) that did not include WISEbased selection, we incorporate a criterion to remove lowredshift quasars from our sample. As seen in Figure 4, the redshift distribution is efficiently truncated for $z<1.5$ compared to the distribution of redshifts from Paper II. 
Of the 108 targets that we observed, we identify 100 quasars and eight stars. However, all (except one) of these eight stars were observed in the first observing run, after which we added a criterion to remove stellar contamination $(J-K>-0.05$ for quasars; see Section 2). After correcting the sample for stellar contamination, we are able to remove six of the eight stars while retaining all the quasars. We therefore have an efficiency of $98 \%$ for selecting quasars. Applying the correction for stellar contamination to the parent sample of 1073 candidates yields 880 high-confidence candidate quasars, of which $232(26 \%)$ are already observed by SDSS and BOSS.

\subsection{Peculiar Quasar Properties}

Similar to our previous surveys presented in Papers I and II, we observe cases where previously parameterized extinction curves (Gordon et al. 2003; Zafar et al. 2015) do not reproduce the observed reddening (see also Hall et al. 2002; Meusinger et al. 2016). In this work, we identify 14 such cases (Section 4.1.2). We illustrate the relative reddening inferred from the data in two cases, and we compare two known reddening laws to the data. While both reddening laws can be made to match the data for a small wavelength range, neither of them can reproduce the full SED. We therefore conclude that not only is the needed reddening law steeper than the SMC (which merely results in a lower absolute extinction $A(V)$ ), but the curvature must also be altered in order to reproduce the apparent breaks in the observed spectra around $\lambda_{\text {rest }} \approx 3000 \AA\left(x \equiv 1 / \lambda \approx 3.5 \mu \mathrm{m}^{-1}\right.$; Figure 5)-the so-called "3000 A break" (Meusinger et al. 2016).

Some part of the mismatch might be ascribed to differences between the assumed template spectrum and the actual intrinsic nature of the quasars in question, e.g., a different slope of the underlying power law of the quasar spectrum. However, a change in the quasar power-law slope would not significantly change the curvature but only the slope of the inferred reddening laws. Similarly, a significant change in the broad emission lines or broad absorption features would show up as strong wiggles in the observed reddening curves instead of the smooth curve that we observe. The small features observed around $x \approx 5 \mu \mathrm{m}^{-1}$ and $x \approx 6 \mu \mathrm{m}^{-1}$ in Figure 5 are indeed due to variations in the emission around C III and C IV, but they are very weak and within the expected variance in these regions (see discussion by Selsing et al. 2016).

Instead, the change in curvature of the reddening law most likely reflects changes in the dust grain properties in the quasars. Such changes could be due to differences in the grain composition or due to a different grain-size distribution. Particularly, a lack of larger grains (typically $0.3 \mu \mathrm{m}$ and larger) leads to steeper and more curved extinction curves (Draine \& Lee 1984; Draine 2003). This can be caused by dust destruction or inhibited grain growth; both are viable explanations in the harsh quasar environments. However, it is then puzzling why we do not observe this type of extinction toward every quasar, as also noted by Leighly et al. (2014). One explanation for this might be that low amounts of reddening effectively mask the curvature (i.e., the noise drowns the shape of the reddening over the full SED). This is especially the case when only a short wavelength range is available. However, as discussed in Goobar (2008) and Leighly et al. (2014), the extinction curve also depends critically on the assumed geometry. Since the dust in the quasar environment is hardly a uniform screen (but more likely distributed all around the central emitting region), the extinction curve can vary significantly due to multiple scattering as the photons propagate through the dusty medium.
Another explanation for the lack of observed curved reddening laws could be due to a clumpy distribution of dust in the surrounding environment where dusty clouds move in and out of the line of sight (Leighly et al. 2015; Netzer 2015). This may explain why this type of dust is generally seen toward highly reddened quasars with large amounts of absorption and not for the regular quasar population, for which little dust is obscuring the view to the central region.

The sample presented by Meusinger et al. (2016) consists of 23 quasars (out of several hundred thousand quasar spectra in SDSS and BOSS) that exhibit the strange $3000 \mathrm{~A}$ break similar to the strange targets identified in this work; see Figure 5. For comparison, we identify six such quasars in our sample of only 100 quasars (when following broadly the same classification criteria for their group A). Moreover, the authors find that the peculiar quasars have a much higher fraction of radio detections $\left(f_{\text {radio }}=74 \%\right)$ than the overall SDSS + BOSS sample $\left(f_{\text {radio }} \sim 8 \%\right.$ [8307/98,544]; Baloković et al. 2012). Meusinger et al. argue that this is most probably due to a selection bias, since quasars in this part of color and redshift space are more frequently targeted for spectroscopic observations based on preexisting radio detections. We observe no radio detections among the six quasars from our survey, consistent with the overall $f_{\text {radio }}$ from SDSS+BOSS.

We inspect the number of targets with radio detections in our candidate sample and find $f_{\text {radio }}=10 \% \pm 1 \%$ (83 out of 880) in our photometric sample. In our spectroscopic sample of 100 quasars, we find a total of seven quasars with radio detections (six from FIRST and one from the NRAO VLA Sky Survey [NVSS]). The fraction of radio detections for the sample presented here is thus fully consistent with the overall $f_{\text {radio }}$ for quasars in SDSS+BOSS. When comparing this to the SDSS + BOSS spectroscopic overlap of our candidates, we observe a much higher fraction of radio detections: $25 \% \pm 3 \%$ of SDSS +BOSS quasars in our candidate sample (57/232) are detected in FIRST. This demonstrates that the SDSS+BOSS sample of quasars, in this part of color space, is biased toward radiobright quasars. It is therefore likely that the high fraction of radio detections observed for the $3000 \AA$ break quasars in Meusinger et al. (2016) is due to this selection artifact and not due to some intrinsic difference in the radio properties of these peculiar quasars. This is further supported by the fact that we observe no enhanced radio detection rate for the six quasars with $3000 \AA$ breaks relative to the parent eHAQ quasars.

While the quasars in our survey are not selected to provide a complete census of the quasar population (we select based on optical properties to look for intervening dust), the spectroscopically identified quasars presented in this survey still provide important observations of the quasar population, which is otherwise sparsely sampled in SDSS and BOSS.

\subsection{Implications for Quasar Selection}

With the advance in recent quasar selection algorithms (e.g., Peters et al. 2015; Richards et al. 2015), new surveys (e.g., eBOSS and LSST) will target the population of red quasars in ever greater detail. Nevertheless, the lack of spectroscopically confirmed high-redshift quasars still limits our ability to model and predict the appearance of quasars. We illustrate this by comparing our sample of quasar candidates (with a purity of around 98\%) to the photometric study by Richards et al. (2015). We cross-match our sample of 880 high-confidence quasars with the photometrically classified quasar catalog of Richards et al. (2015). The matching is performed on the coordinates within a 
matching radius of $1^{\prime}$. Out of 880 eHAQ target candidates, a total of $504(57 \%)$ are recovered in the catalog of Richards et al. (2015). However, this includes 232 quasars from BOSS that made part of the learning sample for their algorithm. Excluding those already-known quasars, we obtain a total of 305 matches out of 648 candidates with no preexisting spectra. Hence, Richards et al. recover $47 \%$ of our candidates. This is also observed in the spectroscopic sample of 100 quasars identified in this work; 47 are recovered in the Richards et al. catalog.

We can compare the number of eHAQ quasars to the total number of quasars selected by Richards et al. in the same area of the sky. We do this by selecting only quasars from the catalog of Richards et al. for which UKIDSS photometry is available, since the smaller extent of the UKIDSS footprint (compared to SDSS and WISE) sets the limit for the available survey area in both studies. ${ }^{12}$ In order to make a fair comparison, we only consider targets with $J_{\mathrm{AB}}<19$ mag (as this makes up the homogenous set of our sample). Moreover, since we reject low-redshift candidates $(z<1.5)$ from eHAQ, we require a similar cut in the photometric redshifts derived by Richards et al. (2015). For this purpose, we use the "ZPHOTBESTJHK" estimate from Richards et al. and impose a conservative redshift limit of $z_{\text {phot }}>1$. Using Table 2 of Richards et al., this yields a total number of 14,641 quasars. Restricting the eHAQ sample to $J_{\mathrm{AB}}<19$ mag yields 588 quasars (4\% of the total number), of which Richards et al. recover about half, i.e., $\sim 2 \%$ of quasars are not identified in the photometric catalog of Richards et al. (2015) down to a limit of $J_{\mathrm{AB}}<19$ mag. However, this fraction might be even larger due to the fact that the eHAQ sample is limited by the inclusion of the shallower bands 3 and 4 from WISE, whereas Richards et al. utilize the deeper AllWISE data for bands 1 and 2 only.

We note that the selection functions of both studies rely on optical and MIR data; however, we include additional NIR data from UKIDSS, which Richards et al. only use for the estimates of photometric redshifts. Much of the difference in candidate selection will therefore be due to the inclusion of NIR data, since most of our ability to separate stars and quasars comes from the $J-K$ color cut, especially for targets with $W_{1}-W_{2}<1$ mag.

Other classification methods involving astrometric data (Koo et al. 1986; Heintz et al. 2015) and time variability data (e.g., Koo et al. 1986; Schmidt et al. 2010; Butler \& Bloom 2011; Graham et al. 2014) will also provide important constraints, although very large temporal baselines are needed to select high-redshift quasars through variability, due to the stretching of observed timescales in an expanding universe. Therefore, in order to push quasar classification to higher completeness (particularly at high redshift), deep NIR large-sky surveys will be of great importance. Selecting quasars in these new ways will expand the population of quasars dramatically; however, spectroscopic confirmation of the candidates is still very timeconsuming, and exploratory surveys like our HAQ survey (Papers I and II) and others (Glikman et al. 2006, 2007, 2012, 2013; Maddox et al. 2008, 2012; Banerji et al. 2013; Hainline et al. 2014) provide important sampling of the parameter space for the advanced classification algorithms.

\subsection{Dusty Absorption Systems}

Only two absorbers are detected in $\operatorname{Ly} \alpha$, both of which are consistent with having $\log N_{\mathrm{H}}>20.3$, but due to the poor

\footnotetext{
12 We remind the reader that we restrict our selection to declinations between $-4^{\circ}$ and $+17^{\circ}$; see Section 2 .
}

spectral quality, it is not possible to determine their column densities precisely. The amount of reddening inferred from the spectral fit for these two Ly $\alpha$ absorbers (eHAQ0930+0148 and eHAQ2359+1354) is $A(V)=0.24$ and $A(V)=0.09$, respectively. Nonetheless, we find no strong evidence for the dust reddening being caused by the absorbers. Thus, we conclude that no dusty DLAs are observed in this survey. However, due to the large fraction of BAL quasars, only 50 (out of a total of 100) quasars are not heavily absorbed by BAL features, which hampers the detection of intervening absorption lines. Of these 50, only 28 are at sufficiently high redshift to detect $\operatorname{Ly} \alpha\left(z_{\mathrm{abs}}>1.8\right)$. Thus, the sample size is too small to draw firm conclusions about the incidence rate of dusty DLAs in this part of color space.

We do, however, detect two absorption systems with evidence for the $2175 \AA$ dust feature. One of these is at too low redshift for us to detect $\operatorname{Ly} \alpha$, and for the other absorber, the Ly $\alpha$ line falls right at the edge of the spectrum, where the noise is very high. We are therefore not able to securely say anything about the column density of $\mathrm{H}$ I for these absorption systems.

For the remaining absorption systems, we do not see any clear evidence for the dust being in the absorber, i.e., we do not see any significant evidence for the $2175 \AA$ bump in the rest frame of the absorption systems. Nevertheless, this cannot be completely ruled out with the current data, since SMC-type extinction in the absorber would be impossible to distinguish from SMC-type dust in the quasar with the limited data available here (e.g., Krogager et al. 2016).

The nondetection of dusty DLAs in our sample shows that these are rare and do not make up a significant part of the population in terms of the incidence rate of neutral hydrogen. However, their importance for studies of cold gas and molecules at high redshift (e.g., Noterdaeme et al. 2007; Srianand et al. 2008; Ledoux et al. 2015; Krogager et al. 2016) still needs to be quantified with larger samples in order to be conclusive. These cold absorbers hold crucial information about the $\mathrm{H}$ I-to- $\mathrm{H}_{2}$ transition and the interplay between dust and gas-phase chemistry (Noterdaeme et al. 2015a, 2016). With more and more complex quasar selection methods, using various wavelength baselines correlated with astrometric and temporal variability data, the discovery of dusty and reddened intervening absorbers will be more frequent due to a more complete parent quasar sample.

We thank the anonymous referee for the constructive comments that helped to improve the quality of this work. The authors thank the many students who dedicated their time to observing quasars during the summer school at the Nordic Optical Telescope in 2015. J.-K.K. acknowledges financial support from the Danish Council for Independent Research (EU-FP7 under the Marie-Curie grant agreement no. 600207) with reference DFF-MOBILEX5051-00115. J.P.U.F. acknowledges support from the ERC-StG grant EGGS-278202. P.N. acknowledges support from the Agence Nationale de la Recherche under grant ANR-12-BS05-0015. M.V. gratefully acknowledges financial support from the Danish Council for Independent Research via grant no. DFF 4002-00275. Based on observations made with the Nordic Optical Telescope, operated on the island of La Palma jointly by Denmark, Finland, Iceland, Norway, and Sweden, in the Spanish Observatorio del Roque de los Muchachos of the Instituto de Astrofísica de Canarias. Funding for SDSS-III has been provided by the Alfred P. Sloan Foundation, the Participating Institutions, the National Science Foundation, and the U.S. Department of Energy Office of Science. The SDSS-III website is http://www.sdss3.org/. SDSS-III is managed by the 
Table 3

Observing Log

\begin{tabular}{|c|c|c|c|c|c|c|}
\hline Target & R.A. (J 2000) & Decl. (J 2000) & $\begin{array}{c}J_{\mathrm{AB}} \\
(\mathrm{mag})\end{array}$ & $\begin{array}{l}\text { Exp. Time } \\
(s)\end{array}$ & Prog. ID ${ }^{\mathrm{a}}$ & Notes \\
\hline eHAQ0001+0431 & 0001042.85 & +043139.05 & 18.16 & $2 \times 600$ & P51-014 & $\ldots$ \\
\hline eHAQ0007+1445 & 0007042.10 & +144528.63 & 17.93 & $2 \times 900$ & P51-802 & $\ldots$ \\
\hline eHAQ0019+0657 & 0019057.28 & +065745.92 & 18.31 & $2 \times 900$ & P51-802 & $\cdots$ \\
\hline eHAQ0026+0708 & 0026011.40 & +070841.32 & 18.08 & $2 \times 600$ & P51-014 & $\ldots$ \\
\hline eHAQ0044+1321 & 0044056.43 & +132148.46 & 17.96 & $2 \times 600$ & P51-014 & $\cdots$ \\
\hline eHAQ0102+1159 & 0102052.91 & +115948.40 & 18.51 & $1 \times 900$ & P51-802 & BOSS overlap \\
\hline eHAQ0104+0756 & 0104017.06 & +075635.20 & 17.65 & $2 \times 600$ & P51-014 & $\cdots$ \\
\hline eHAQ0104+0912 & 0104011.72 & +09 1238.49 & 18.38 & $2 \times 600$ & P51-014 & $\cdots$ \\
\hline eHAQ0104+1506 & 0104041.53 & +150626.40 & 17.91 & $2 \times 1000$ & INT-C165 & Isaac Newton Telescope \\
\hline eHAQ0109+0435 & 0109011.23 & +043544.22 & 17.65 & $2 \times 900$ & P51-014 & $\ldots$ \\
\hline eHAQ0111+0641 & 0111034.71 & +064119.22 & 18.57 & $2 \times 900$ & P51-014 & $\cdots$ \\
\hline eHAQ0113+0804 & 0113055.39 & +080425.68 & 17.30 & $2 \times 600$ & P51-014 & $\cdots$ \\
\hline eHAQ0121+1028 & 0121034.45 & +102834.18 & 19.28 & $2 \times 1200$ & P51-014 & $\cdots$ \\
\hline eHAQ0129+0638 & 0129050.61 & +063846.00 & 18.02 & $2 \times 1300$ & P51-014 & $\cdots$ \\
\hline eHAQ0129+1039 & 0129018.99 & +103943.16 & 19.07 & $2 \times 1200$ & P51-014 & $\ldots$ \\
\hline eHAQ0138+0742 & 0138014.45 & +074235.49 & 18.35 & $2 \times 600$ & P51-014 & $\ldots$ \\
\hline eHAQ0142+0257 & 0142006.88 & +025713.06 & 16.68 & $2 \times 600$ & P51-014 & $\cdots$ \\
\hline eHAQ0147+0411 & 0147032.03 & +041113.41 & 17.42 & $2 \times 700$ & INT-C165 & Isaac Newton Telescope \\
\hline eHAQ0147+1549 & 0147016.89 & +154943.99 & 17.20 & $2 \times 700$ & P51-014 & $\ldots$ \\
\hline eHAQ0157+1321 & 0157001.54 & +132118.83 & 17.89 & $2 \times 1200$ & INT-C165 & Isaac Newton Telescope \\
\hline eHAQ0216+0426 & 0216043.27 & +042628.75 & 18.81 & $2 \times 1200$ & P51-014 & $\ldots$ \\
\hline eHAQ0227+0521 & 0227057.93 & +052141.88 & 18.40 & $1 \times 600$ & P51-014 & $\cdots$ \\
\hline eHAQ0300+0440 & 0300002.47 & +044004.89 & 18.54 & $2 \times 600$ & P51-014 & $\cdots$ \\
\hline eHAQ0321+0523 & 0321052.20 & +052337.40 & 17.91 & $1 \times 900$ & P51-014 & BOSS overlap \\
\hline eHAQ0347+0348 & 0347034.17 & +034836.88 & 18.41 & $2 \times 600$ & P51-014 & $\ldots$ \\
\hline eHAQ0828+0313 & 0828013.98 & +031354.35 & 17.21 & $3 \times 200$ & P50-802 & $\cdots$ \\
\hline eHAQ0835+0127 & 0835015.82 & +012731.42 & 18.00 & $3 \times 600$ & P50-802 & $J-K<-0.05$ \\
\hline eHAQ0835+0830 & 0835042.19 & +08 3010.37 & 16.75 & $3 \times 200$ & P50-802 & $J-K<-0.05$ \\
\hline eHAQ0839+0556 & 0839002.87 & +055627.77 & 18.03 & $3 \times 500$ & P50-802 & $\cdots$ \\
\hline eHAQ0852+0204 & 0852003.84 & +020437.79 & 18.41 & $3 \times 900$ & P52-017 & $\ldots$ \\
\hline eHAQ0856+0643 & 0856028.31 & +064354.18 & 18.67 & $3 \times 900$ & P50-802 & $\ldots$ \\
\hline eHAQ0913+0910 & 0913004.43 & +091044.24 & 17.59 & $3 \times 250$ & P50-802 & $\ldots$ \\
\hline eHAQ0915+0115 & 0915035.57 & +011515.43 & 17.38 & $3 \times 400$ & P50-802 & $J-K<-0.05$ \\
\hline eHAQ0919+0843 & 0919007.11 & +084305.04 & 18.38 & $1 \times 900$ & P52-017 & $\ldots$ \\
\hline eHAQ0921+0149 & 0921042.04 & +014959.75 & 17.70 & $3 \times 900$ & P50-802 & $\cdots$ \\
\hline eHAQ0923+0520 & 0923045.76 & +05 2026.41 & 17.79 & $3 \times 600$ & P50-802 & $\ldots$ \\
\hline eHAQ0927-0233 & 0927040.04 & -023347.47 & 18.95 & $3 \times 900$ & P52-017 & $\ldots$ \\
\hline eHAQ0930+0148 & 0930045.09 & +014846.69 & 18.69 & $3 \times 900$ & P52-017 & $\cdots$ \\
\hline eHAQ0940+0532 & 0940051.36 & +053213.29 & 17.67 & $3 \times 500$ & P50-802 & $\ldots$ \\
\hline eHAQ0943+0954 & 0943049.65 & +095400.93 & 17.88 & $3 \times 600$ & P50-802 & BOSS overlap \\
\hline eHAQ0943+1300 & 0943032.99 & +1300 55.74 & 17.91 & $3 \times 600$ & P52-017 & $\ldots$ \\
\hline eHAQ0949+1207 & 0949046.98 & +120756.16 & 18.53 & $2 \times 600$ & P52-017 & $\ldots$ \\
\hline eHAQ0950+0440 & 0950004.09 & +044040.35 & 18.60 & $3 \times 600$ & P52-017 & $\ldots$ \\
\hline eHAQ0952+0835 & 0952002.99 & +083555.84 & 18.12 & $3 \times 900$ & P52-017 & $\ldots$ \\
\hline eHAQ1002+0406 & 1002051.28 & +040653.95 & 16.42 & $3 \times 400$ & P50-802 & $J-K<-0.05$ \\
\hline eHAQ1005+0602 & 1005001.82 & +060219.88 & 18.41 & $2 \times 900$ & P52-017 & $\ldots$ \\
\hline eHAQ1010+1158 & 1010054.03 & +115805.70 & 18.10 & $3 \times 600$ & P50-802 & $\cdots$ \\
\hline eHAQ1025+1324 & 1025018.62 & +132412.05 & 17.53 & $3 \times 500$ & P50-802 & $\ldots$ \\
\hline eHAQ1026-0241 & 1026000.91 & -024117.08 & 18.34 & $2 \times 900$ & P52-017 & $\ldots$ \\
\hline eHAQ1030+1040 & 1030032.55 & +104051.34 & 17.25 & $3 \times 200$ & P50-802 & $\cdots$ \\
\hline eHAQ1106+0844 & 1106035.85 & +084402.35 & 18.44 & $3 \times 900$ & P50-802 & BOSS overlap \\
\hline eHAQ1109+0135 & 1109002.31 & +013538.81 & 16.62 & $3 \times 400$ & P50-802 & $J-K<-0.05$ \\
\hline eHAQ1109+1058 & 1109024.83 & +105825.43 & 18.82 & $2 \times 900$ & P52-017 & $\ldots$ \\
\hline eHAQ1111+0151 & 1111037.69 & +015147.27 & 17.07 & $3 \times 360$ & P50-802 & $J-K<-0.05$ \\
\hline eHAQ1119+1430 & 1119039.72 & +143000.76 & 18.43 & $3 \times 600$ & P52-017 & $\ldots$ \\
\hline eHAQ1120+0812 & 1120003.66 & +081212.02 & 15.57 & $3 \times 100$ & P52-017 & $\ldots$ \\
\hline eHAQ1132+1243 & 1132007.19 & +124342.09 & 17.32 & $3 \times 600$ & P50-802 & $\cdots$ \\
\hline eHAQ1136+0027 & 1136044.12 & +002700.30 & 18.12 & $3 \times 900$ & P52-017 & $\cdots$ \\
\hline eHAQ1144+0902 & 1144045.44 & +0902 26.70 & 17.92 & $3 \times 600$ & P50-802 & BOSS overlap \\
\hline eHAQ1202+0423 & 1202004.85 & +042341.00 & 18.57 & $2 \times 600$ & P52-017 & $\ldots$ \\
\hline eHAQ1203+0652 & 1203036.93 & +065234.47 & 18.91 & $3 \times 900$ & P52-017 & $\ldots$ \\
\hline eHAQ1203+1118 & 1203033.96 & +111826.02 & 18.00 & $3 \times 900$ & P50-802 & $\ldots$ \\
\hline eHAQ1210+1429 & 1210020.99 & +142958.53 & 16.10 & $3 \times 200$ & P50-802 & $\ldots$ \\
\hline
\end{tabular}


Table 3

(Continued)

\begin{tabular}{|c|c|c|c|c|c|c|}
\hline Target & R.A. (J 2000) & Decl. (J 2000) & $\begin{array}{c}J_{\mathrm{AB}} \\
(\mathrm{mag})\end{array}$ & $\begin{array}{l}\text { Exp. Time } \\
(s)\end{array}$ & Prog. ID ${ }^{\mathrm{a}}$ & Notes \\
\hline eHAQ1222+0826 & 1222014.97 & +082610.15 & 18.69 & $2 \times 900$ & P52-017 & $\ldots$ \\
\hline eHAQ1226-0236 & 1226055.31 & -023657.90 & 18.63 & $3 \times 900$ & P52-017 & $\ldots$ \\
\hline eHAQ1237+1233 & 1237026.47 & +123350.23 & 18.71 & $2 \times 900$ & P52-017 & $\ldots$ \\
\hline eHAQ1244+0841 & 1244018.82 & +084136.10 & 18.32 & $3 \times 800$ & P50-802 & $\ldots$ \\
\hline eHAQ1252+0842 & 1252057.73 & +084206.06 & 17.93 & $3 \times 600$ & P50-802 & $\ldots$ \\
\hline eHAQ1312+1431 & 1312014.18 & +143111.28 & 18.53 & $2 \times 900$ & P52-017 & $\ldots$ \\
\hline eHAQ1326+1317 & 1326024.77 & +131727.52 & 17.64 & $6 \times 300$ & P50-802 & BOSS overlap \\
\hline eHAQ1331+1304 & 1331029.90 & +130420.90 & 17.11 & $3 \times 600$ & P50-802 & $J-K<-0.05$ \\
\hline eHAQ1340+0151 & 1340044.56 & +015141.44 & 18.16 & $4 \times 300$ & P52-017 & $\ldots$ \\
\hline eHAQ1340+1458 & 1340053.91 & +145853.81 & 17.80 & $2 \times 900$ & P52-017 & $\ldots$ \\
\hline eHAQ1346+0114 & 1346032.37 & +011408.38 & 18.27 & $3 \times 900$ & P50-802 & $\ldots$ \\
\hline eHAQ1357-0051 & 1357030.57 & -005141.09 & 16.60 & $3 \times 300$ & P52-017 & $\ldots$ \\
\hline eHAQ1400+0720 & 1400039.19 & +072011.99 & 18.38 & $2 \times 900$ & P52-017 & $\ldots$ \\
\hline eHAQ1447+0521 & 1447021.61 & +052141.70 & 18.54 & $3 \times 900$ & P52-017 & $\ldots$ \\
\hline eHAQ1450+1002 & 1450043.69 & +100238.79 & 18.38 & $2 \times 900$ & P52-017 & $\ldots$ \\
\hline eHAQ1455+0705 & 1455010.53 & +070525.50 & 16.39 & $3 \times 200$ & P50-802 & $\ldots$ \\
\hline eHAQ1514-0002 & $15 \quad 14001.87$ & -000259.66 & 18.31 & $3 \times 600$ & P52-017 & $\ldots$ \\
\hline eHAQ1525+0155 & 1525055.01 & +015513.54 & 18.25 & $3 \times 900$ & P52-017 & $\cdots$ \\
\hline eHAQ1528+0546 & 1528053.64 & +054657.42 & 17.99 & $6 \times 360$ & P50-802 & BOSS overlap \\
\hline eHAQ1539+0351 & 1539035.25 & +035125.57 & 17.00 & $3 \times 360$ & P50-802 & $\ldots$ \\
\hline eHAQ1543+0447 & 1543027.29 & +044717.75 & 17.96 & $2 \times 900$ & P52-017 & $\ldots$ \\
\hline eHAQ1549+0501 & 1549036.57 & +050128.04 & 18.11 & $3 \times 900$ & P52-017 & $\ldots$ \\
\hline eHAQ2209+0304 & 2209020.36 & +030436.02 & 18.24 & $2 \times 900$ & P51-802 & $\cdots$ \\
\hline eHAQ2235+0635 & 2235012.40 & +063545.49 & 18.11 & $2 \times 400$ & P51-014 & $\ldots$ \\
\hline eHAQ2236+0731 & 2236009.38 & +073108.20 & 19.08 & $2 \times 900$ & P51-014 & $\ldots$ \\
\hline eHAQ2247+0922 & 2247009.08 & +092233.46 & 18.46 & $2 \times 900$ & P51-802 & $\ldots$ \\
\hline eHAQ2255+1213 & 2255058.75 & +121310.91 & 18.73 & $2 \times 600$ & P51-014 & $\ldots$ \\
\hline eHAQ2256+0531 & 2256043.90 & +053115.07 & 18.65 & $2 \times 1500$ & P51-014 & $\ldots$ \\
\hline eHAQ2258+0251 & 2258043.01 & +025107.21 & 19.42 & $2 \times 1500$ & P51-014 & $\cdots$ \\
\hline eHAQ2259+0208 & 2259055.76 & +020825.39 & 18.10 & $2 \times 400$ & P51-014 & $\cdots$ \\
\hline eHAQ2259+0256 & 2259020.36 & +025643.76 & 17.80 & $2 \times 600$ & P51-014 & $\ldots$ \\
\hline eHAQ2259+0736 & 2259017.85 & +073633.47 & 18.30 & $2 \times 1000$ & P51-802 & $\ldots$ \\
\hline eHAQ2301+0752 & 2301059.41 & +075238.95 & 18.78 & $2 \times 900$ & P51-014 & $\ldots$ \\
\hline eHAQ2303+0747 & 2303000.30 & +074736.78 & 18.34 & $2 \times 1080$ & INT-C165 & Isaac Newton Telescope \\
\hline eHAQ2309+1159 & 2309014.78 & +115955.99 & 18.25 & $2 \times 900$ & P51-014 & $\cdots$ \\
\hline eHAQ2310+0447 & 2310043.96 & +044746.77 & 18.94 & $2 \times 900$ & P51-802 & $\cdots$ \\
\hline eHAQ2313+1259 & 2313024.39 & +125939.01 & 18.32 & $2 \times 900$ & P51-014 & $\ldots$ \\
\hline eHAQ2314+0552 & $23 \quad 14053.38$ & +055233.11 & 18.95 & $2 \times 1000$ & P51-014 & $\ldots$ \\
\hline eHAQ2316+0651 & 2316007.06 & +065145.09 & 18.90 & $2 \times 1000$ & P51-014 & $\ldots$ \\
\hline eHAQ2321+1107 & 2321051.29 & +110720.67 & 17.01 & $2 \times 300$ & P51-014 & $\cdots$ \\
\hline eHAQ2321+1121 & 2321052.27 & +112128.50 & 19.11 & $2 \times 1000$ & P51-014 & $\ldots$ \\
\hline eHAQ2334+1519 & 2334021.51 & +151947.56 & 18.60 & $2 \times 1200$ & P51-014 & $\ldots$ \\
\hline eHAQ2344+1416 & 2344051.94 & +141640.46 & 17.59 & $2 \times 500$ & P51-014 & $\ldots$ \\
\hline eHAQ2358+1030 & 2358040.47 & +103039.94 & 18.28 & $2 \times 1200$ & P51-014 & $\cdots$ \\
\hline eHAQ2359+1354 & 2359016.50 & +135443.35 & 18.92 & $2 \times 800$ & P51-802 & Grism \#6, BOSS overlap \\
\hline
\end{tabular}

Note.

a Observing program identifier. If nothing else is stated, the observations were carried out at the Nordic Optical Telescope.

Astrophysical Research Consortium for the Participating Institutions of the SDSS-III Collaboration, including the University of Arizona, the Brazilian Participation Group, Brookhaven National Laboratory, Carnegie Mellon University, University of Florida, the French Participation Group, the German Participation Group, Harvard University, the Instituto de Astrofísica de Canarias, the Michigan State/Notre Dame/JINA Participation Group, Johns Hopkins University, Lawrence Berkeley National Laboratory, Max Planck Institute for Astrophysics, Max Planck Institute for Extraterrestrial Physics, New Mexico State University, New York University, Ohio State University, Pennsylvania State University, University of Portsmouth, Princeton University, the Spanish Participation Group, University of Tokyo, University of Utah,
Vanderbilt University, University of Virginia, University of Washington, and Yale University. This publication makes use of data products from the Wide-field Infrared Survey Explorer, which is a joint project of the University of California, Los Angeles, and the Jet Propulsion Laboratory/California Institute of Technology, funded by the National Aeronautics and Space Administration.

\section{APPENDIX A TABLES}

The details of the observations are summarized in Table 3. Results from the spectral classification and dust fitting are given in Table 4. 
Table 4

Quasar Classification

\begin{tabular}{|c|c|c|c|}
\hline Target & $z$ & $\begin{array}{l}A(V) \\
(\mathrm{mag})\end{array}$ & Classification \\
\hline eHAQ0001+0431 & 1.96 & $0.52 \pm 0.01$ & Quasar \\
\hline eHAQ0007+1445 & 1.37 & $0.68 \pm 0.01$ & BAL quasar \\
\hline eHAQ0019+0657 & 3.37 & $0.17 \pm 0.01$ & Quasar \\
\hline eHAQ0026+0708 & 1.78 & $0.50 \pm 0.01$ & Quasar \\
\hline eHAQ0044+1321 ${ }^{\mathrm{a}}$ & 1.70 & $0.16 \pm 0.01$ & BAL quasar \\
\hline eHAQ0102+1159 & 4.25 & $0.15 \pm 0.03$ & BAL quasar \\
\hline eHAQ0104+0756 ${ }^{\mathrm{a}}$ & 2.21 & $0.47 \pm 0.01$ & BAL quasar \\
\hline eHAQ0104+0912 & 2.01 & $0.22 \pm 0.01$ & Quasar \\
\hline eHAQ0104+1506 & 1.42 & $0.3-0.6$ & Quasar \\
\hline eHAQ0109+0435 & 3.47 & $0.20 \pm 0.05$ & BAL quasar \\
\hline eHAQ0111+0641 & 3.23 & $0.17 \pm 0.01$ & Quasar \\
\hline eHAQ0113+0804 & 1.97 & $0.60 \pm 0.01$ & BAL quasar \\
\hline eHAQ0121+1028 & 2.10 & $0.63 \pm 0.03$ & Quasar \\
\hline eHAQ0129+0638 & 2.40 & $0.52 \pm 0.01$ & BAL quasar \\
\hline eHAQ0129+1039 & 0.57 & $1.23 \pm 0.02$ & Weak line quasar \\
\hline eHAQ0138+0742 & 3.21 & $0.14 \pm 0.03$ & BAL quasar \\
\hline $\mathrm{eHAQ} 0142+0257^{\mathrm{a}}$ & 2.30 & $0.38 \pm 0.01$ & BAL quasar \\
\hline eHAQ0147+0411 & 1.77 & $0.37 \pm 0.01$ & Quasar \\
\hline eHAQ0147+1549 & $\cdots$ & $\ldots$ & BL Lacertae Object \\
\hline eHAQ0157+1321 & 1.73 & $0.44 \pm 0.02$ & Quasar \\
\hline eHAQ0216+0426 & 1.58 & $0.60 \pm 0.01$ & Quasar \\
\hline eHAQ0227+0521 & 2.68 & $0.14 \pm 0.02$ & BAL quasar \\
\hline eHAQ0300+0440 a & 1.88 & $0.30 \pm 0.01^{\mathrm{b}}$ & Quasar \\
\hline eHAQ0321+0523 & 2.97 & $0.20 \pm 0.01$ & BAL quasar \\
\hline eHAQ0347+0348 & 1.85 & $0.19 \pm 0.01$ & Quasar \\
\hline eHAQ0828+0313 & 1.99 & $0-0.27$ & Weak line quasar \\
\hline eHAQ0835+0127 & 3.30 & $0.14 \pm 0.01$ & Quasar \\
\hline eHAQ0839+0556 ${ }^{\mathrm{a}}$ & 1.49 & $0.64 \pm 0.10^{\mathrm{b}}$ & Weak line quasar \\
\hline eHAQ0852+0204 & 2.20 & $0.58 \pm 0.01$ & BAL quasar \\
\hline eHAQ0856+0643 ${ }^{\mathrm{a}}$ & 2.49 & $0-0.44$ & Quasar \\
\hline eHAQ0913+0910 & 1.95 & $0.00 \pm 0.01$ & BAL quasar \\
\hline eHAQ0919+0843 & 2.43 & $0.35 \pm 0.05$ & BAL quasar \\
\hline eHAQ0921+0149 & 0.99 & $1.06 \pm 0.01$ & Quasar \\
\hline eHAQ0923+0520 & 3.39 & $0.12 \pm 0.06$ & BAL quasar \\
\hline eHAQ0927-0233 & 1.24 & $0.72 \pm 0.02$ & Quasar \\
\hline eHAQ0930+0148 & 2.95 & $0.24 \pm 0.01$ & Quasar \\
\hline eHAQ0940+0532 & 2.32 & $0.19 \pm 0.05$ & Weak line quasar \\
\hline eHAQ0943+0954 & 4.18 & $0.00 \pm 0.01$ & BAL quasar \\
\hline eHAQ0943+1300 & 2.68 & $0.18 \pm 0.11$ & BAL quasar \\
\hline eHAQ0949+1207 & 3.36 & $0.03 \pm 0.01$ & BAL quasar \\
\hline eHAQ0950+0440 a & 2.30 & $0-0.53$ & BAL quasar \\
\hline eHAQ0952+0835 & 1.86 & $0.64 \pm 0.01$ & BAL quasar \\
\hline eHAQ1005+0602 & 2.57 & $0.35 \pm 0.01$ & BAL quasar \\
\hline eHAQ1010+1158 & 2.24 & $0-0.40$ & BAL quasar \\
\hline eHAQ1025+1324 & 2.57 & $0.15-0.78$ & BAL quasar \\
\hline eHAQ1026-0241 & 3.23 & $0.24 \pm 0.01$ & Quasar \\
\hline eHAQ1030+1040 & 1.58 & $0-0.50$ & BAL quasar \\
\hline eHAQ1106+0844 & 1.86 & $0.37 \pm 0.01$ & BAL quasar \\
\hline eHAQ1109+1058 & 1.90 & $0.43 \pm 0.01$ & Quasar \\
\hline eHAQ1119+1430 & 1.88 & $0.31 \pm 0.01$ & BAL quasar \\
\hline eHAQ1136+0027 & 1.12 & $0.95 \pm 0.02$ & Quasar \\
\hline eHAQ1144+0902 & 1.78 & $0.33 \pm 0.01$ & BAL quasar \\
\hline eHAQ1202+0423 & 2.32 & $0.02 \pm 0.01$ & BAL quasar \\
\hline eHAQ1203+0652 & 2.40 & $0.26 \pm 0.03$ & BAL quasar \\
\hline eHAQ1203+1118 & 3.62 & $0.15 \pm 0.02$ & BAL quasar \\
\hline eHAQ1210+1429a & 1.48 & $0.36-1.10$ & Weak line quasar \\
\hline eHAQ1222+0826 & 2.90 & $0.22 \pm 0.02$ & BAL quasar \\
\hline eHAQ1226-0236 & 1.25 & $0.77 \pm 0.01$ & Quasar \\
\hline $\mathrm{eHAQ} 1237+1233^{\mathrm{a}}$ & 2.31 & $0.12 \pm 0.03$ & BAL quasar \\
\hline eHAQ1244+0841 & 1.87 & $0.25-0.45$ & Quasar \\
\hline eHAQ1252+0842 & 3.53 & $0.07 \pm 0.01$ & BAL quasar \\
\hline eHAQ1312+1431 & 1.64 & $0.51 \pm 0.01$ & Quasar \\
\hline eHAQ1326+1317 & 2.00 & $0.00 \pm 0.01$ & BAL quasar \\
\hline
\end{tabular}

Table 4

(Continued)

\begin{tabular}{lccl}
\hline \hline Target & $z$ & $\begin{array}{c}A(V) \\
(\mathrm{mag})\end{array}$ & Classification \\
\hline eHAQ1340+1458 $^{\mathrm{a}}$ & 1.73 & $0.3-0.61$ & Quasar \\
eHAQ1340+0151 $^{\mathrm{a}}$ & 1.94 & $0-0.94$ & $\begin{array}{l}\text { Tentative identification; no } \\
\text { spectral features }\end{array}$ \\
& & & Quasar \\
eHAQ1346+0114 $^{\mathrm{a}}$ & 1.73 & $0.3-1.10$ & BAL quasar \\
eHAQ1357-0051 & 1.80 & $0.71 \pm 0.01$ & BAL \\
eHAQ1400+0720 & 2.40 & $0.38 \pm 0.02$ & BAL quasar \\
eHAQ1447+0521 & 2.35 & $0.51 \pm 0.02$ & BAL quasar \\
eHAQ1450+1002 & 1.58 & $0.36 \pm 0.01$ & BAL quasar \\
eHAQ1455+0705 & 1.90 & $0.52 \pm 0.01$ & Quasar \\
eHAQ1514-0002 & 1.70 & $0.00 \pm 0.01$ & BAL quasar \\
eHAQ1525+0155 & 1.62 & $0.74 \pm 0.01$ & BAL quasar \\
eHAQ1528+0546 & 2.12 & $0.38 \pm 0.01$ & Quasar \\
eHAQ1539+0351 & 1.63 & $0.29 \pm 0.01$ & BAL quasar \\
eHAQ1543+0447 & 2.05 & $0.71 \pm 0.01$ & Quasar \\
eHAQ1549+0501 & 1.46 & $0.74 \pm 0.01$ & Quasar \\
eHAQ2209+0304 & 2.33 & $0.35 \pm 0.01$ & Quasar \\
eHAQ2235+0635 & 1.13 & $0.81 \pm 0.01$ & Quasar \\
eHAQ2236+0731 & 1.72 & $0.26 \pm 0.01$ & Quasar \\
eHAQ2247+0922 & 1.63 & $0.56 \pm 0.02$ & Quasar \\
eHAQ2255+1213 & 2.05 & $0.14 \pm 0.01$ & Quasar \\
eHAQ2256+0531 & 1.35 & $0.88 \pm 0.03$ & Quasar \\
eHAQ2258+0251 & 2.43 & $0.40 \pm 0.03$ & BAL quasar \\
eHAQ2259+0208 & 2.05 & $0.36 \pm 0.01$ & Quasar \\
eHAQ2259+0256 & 1.83 & $0.59 \pm 0.01$ & Quasar \\
eHAQ2259+0736 & 0.78 & $1.23 \pm 0.02$ & Quasar \\
eHAQ2301+0752 & 2.62 & $0.27 \pm 0.02$ & BAL quasar \\
eHAQ2303+0747 & 1.31 & $0.57 \pm 0.04$ & Quasar \\
eHAQ2309+1159 & 2.00 & $0.54 \pm 0.01$ & Quasar \\
eHAQ2310+0447 & 1.42 & $0.42 \pm 0.01$ & BAL quasar \\
eHAQ2313+1259 & 2.11 & $0.57 \pm 0.01$ & Quasar \\
eHAQ2314+0552 & 2.03 & $0.27 \pm 0.01$ & BAL quasar \\
eHAQ2316+0651 & 1.37 & $0.53 \pm 0.01$ & BAL quasar \\
eHAQ2321+1107 & 1.56 & $0.54 \pm 0.01$ & BAL quasar \\
eHAQ2321+1121 & 2.18 & $0.37 \pm 0.01$ & Quasar \\
eHAQ2334+1519 & 2.23 & $0.55 \pm 0.01$ & BAL quasar \\
eHAQ2344+1416 & 2.52 & $0.41 \pm 0.02$ & BAL quasar \\
eHAQ2358+1030 & 2.05 & $1.03 \pm 0.02$ & Quasar \\
eHAQ2359+1354 & 2.79 & $0.13 \pm 0.03^{\mathrm{c}}$ & Quasar \\
\hline & & &
\end{tabular}

Notes. Uncertainties given on $A(V)$ are only statistical errors from the fit. Where we give a range in $A(V)$, the first and last numbers indicate the amount of reddening inferred from the NIR and optical data, respectively. The classification of BALs is done by eye and does not follow a rigorous scheme.

a Target is classified as "peculiar" quasar; see text.

b Assuming extinction curve from Zafar et al. (2015).

${ }^{\mathrm{c}}$ Determined from the BOSS spectrum.

\section{APPENDIX B STELLAR CONTAMINANTS}

The individual spectral classifications of stellar contaminants are given in Table 5. Their spectra and broadband photometry are shown in Figure 7.

\section{APPENDIX C INDIVIDUAL FITS FOR DUST IN INTERVENING ABSORBERS}

In Figure 8, the spectra and broadband photometry are shown together with the best-fit dust models for the four cases where an intervening system has been identified statistically. The best-fit parameters are summarized in Table 6 . 
Table 5

Stellar Classification

\begin{tabular}{ll}
\hline \hline Target & Spectral Type \\
\hline eHAQ0835+0830 & K4 v \\
eHAQ0915+0115 & K5 v \\
eHAQ1002+0406 & M2 V \\
eHAQ1109+0135 & M2 V \\
eHAQ1111+0151 & K7 v \\
eHAQ1120+0812 & K5 III \\
eHAQ1132+1243 & K4 III (metal-rich); tentative \\
eHAQ1331+1304 & M1 V \\
\hline
\end{tabular}

Note. The spectral types have been determined from a template-matching algorithm.

\section{APPENDIX D NOTES ON INDIVIDUAL OBJECTS}

\section{D.1. eHAQ0001+0431 $(z=1.96)$}

This is a reddened quasar with weak but broad absorption blueward of $\mathrm{C} \mathrm{III]}$.

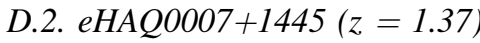

This is a reddened quasar with narrow associated absorption at the redshift of the quasar and broad absorption blueward of $\mathrm{C}$ III] and $\mathrm{Mg}$ II with varying relative velocity offsets.

\section{D.3. eHAQ0019+0657 $(z=3.37)$}

This is a reddened quasar with an intervening $\mathrm{Mg}$ II absorption system at $z_{\mathrm{abs}}=1.672$.

$$
\text { D.4. eHAQ0026+0708 }(z=1.78)
$$

This is a reddened quasar with weak absorption around C IV.

$$
\text { D.5. eHAQ0044+1321 }(z=1.70)
$$

This is a highly absorbed BAL quasar with weak emission lines. Due to the large amount of intrinsic absorption, the template is a very poor match to the observed optical spectrum. The reddening is inferred from the infrared photometry only.

$$
\text { D.6. } \mathrm{eHAQ0102+1159(z=4.25)}
$$

This is a quasar with broad absorption blueward of Si IV. The Lyman break from the associated absorption at the redshift of the quasar is observed around $\lambda_{\text {obs }} \approx 4800 \AA$.

$$
\text { D.7. } e H A Q 0104+0756(z=2.21)
$$

This is an FeLoBAL quasar. The reddening is roughly estimated from the photometry. However, due to the high level of absorption, it is impossible to determine the intrinsic continuum level.

\section{D.8. eHAQ0104+0912 $(z=2.01)$}

This is a reddened quasar with an intervening $\mathrm{Mg}$ II absorption system at $z_{\mathrm{abs}}=1.487$. There is absorption around $\mathrm{C}$ IV and C III], which is consistent with the presence of the $2175 \AA$ bump at the redshift of the absorber.

$$
\text { D.9. } \mathrm{eHAQ0104+1506}(z=1.42)
$$

The dust fitting is insecure due to flux calibration issues of the INT spectrum. The Mg II is partly absorbed by associated absorption. The Fe II emission around the $\mathrm{Mg}$ II emission line is possibly very strong, causing the $\mathrm{Mg}$ II line to appear weaker. The optical data favor a solution with high dust extinction $(A$ $(V) \approx 0.6)$, whereas the NIR data indicate somewhat lower extinction $(A(V) \approx 0.3)$. The optical and NIR data might be reconciled by a steeper extinction curve or a steeper intrinsic power-law slope, or a combination of both.

$$
\text { D.10. eHAQ0109+0435 }(z=3.47)
$$

This is a reddened BAL quasar with very weak emission lines and a very complex absorption-line structure. The redshift is determined from absorption lines and from the small emission line around $\lambda_{\text {obs }} \approx 4800 \AA$, which is interpreted as Ly $\beta$. The absolute flux calibration is insecure due to the scaling to the $r$ band, which is highly affected by the BAL features. The reddening is therefore only estimated from the photometry. There is possibly an excess of Fe II emission causing the observed excess in the $z$ and $Y$ bands compared to the best-fit template.

\section{D.11. eHAQ0111+0641 $(z=3.23)$}

This is a reddened quasar with an intervening absorption system at $z_{\mathrm{abs}}=2.027$ determined from Fe II lines since $\mathrm{Mg}$ II is just outside the spectral coverage.

$$
\text { D.12. eHAQ0113+0804 }(z=1.97)
$$

This is a reddened BAL quasar with associated metal absorption from Fe II and $\mathrm{Mg}$ II at $z=1.938$. Furthermore, there is an intervening absorption system at $z_{\mathrm{abs}}=1.589$.

$$
\text { D.13. eHAQ0121+1028 }(z=2.10)
$$

This is a reddened quasar.

$$
\text { D.14. eHAQ0129+0638 }(z=2.40)
$$

This is a reddened BAL quasar with associated iron absorption.

$$
\text { D.15. eHAQ0129+1039 }(z=0.567)
$$

This is a highly reddened low-redshift active galactic nuclues with weak broad lines. The infrared excess in the observed $H$ and $K$ bands is most likely caused by a combination of host galaxy emission and hot dust.

$$
\text { D.16. eHAQ0138+0742 }(z=3.21)
$$

This is a reddened quasar with narrow associated absorption. The Lyman break from the associated absorber is visible in the blue edge of the spectrum.

$$
\text { D.17. eHAQ0142+0257 }(z=2.30)
$$

This is a reddened quasar with narrow associated absorption especially in C IV, Si IV, and N V. The strong absorption around $\mathrm{CIV}$ and $\mathrm{CIII}]$ is possibly caused by absorption of the iron "pseudo-continuum." The object is detected in both FIRST and NVSS (Condon et al. 1998) at $1.4 \mathrm{GHz}$. 


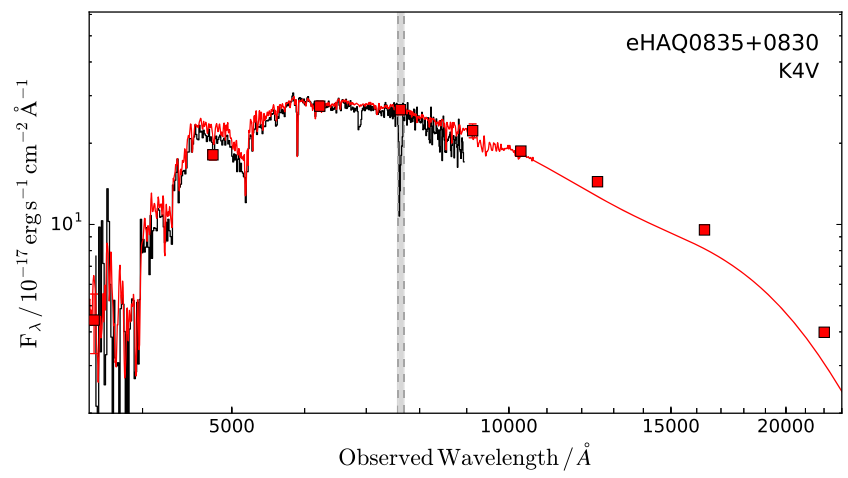

(a)

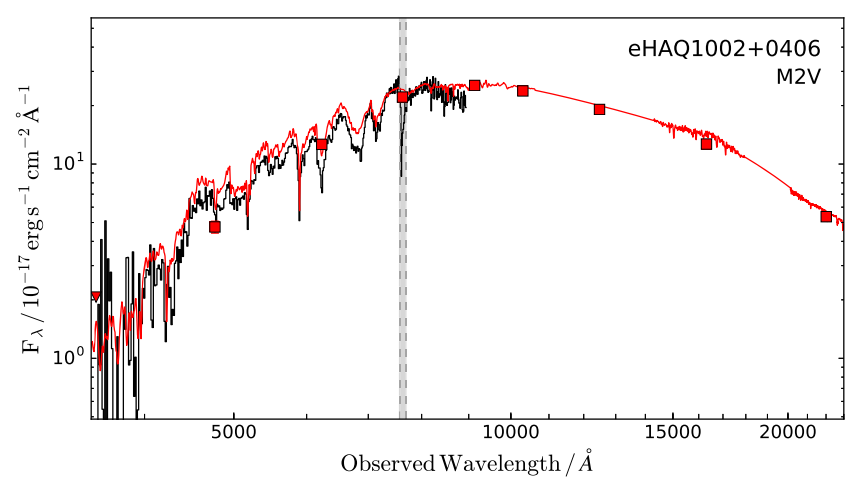

(c)

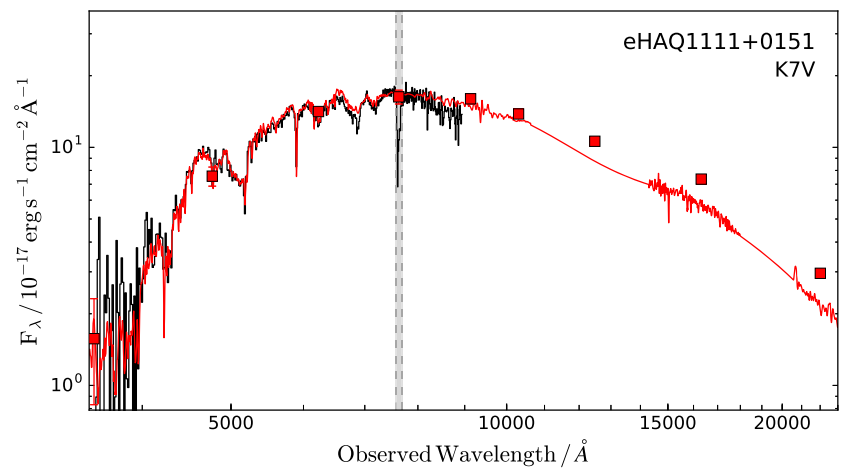

(e)

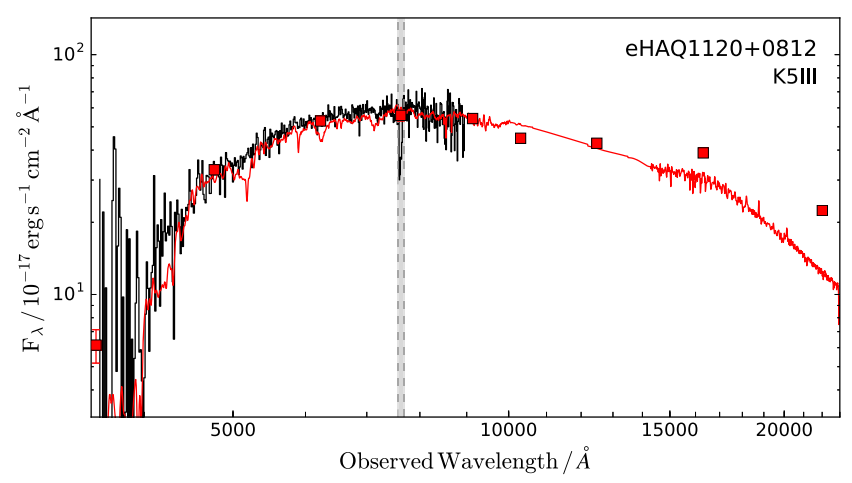

(g)

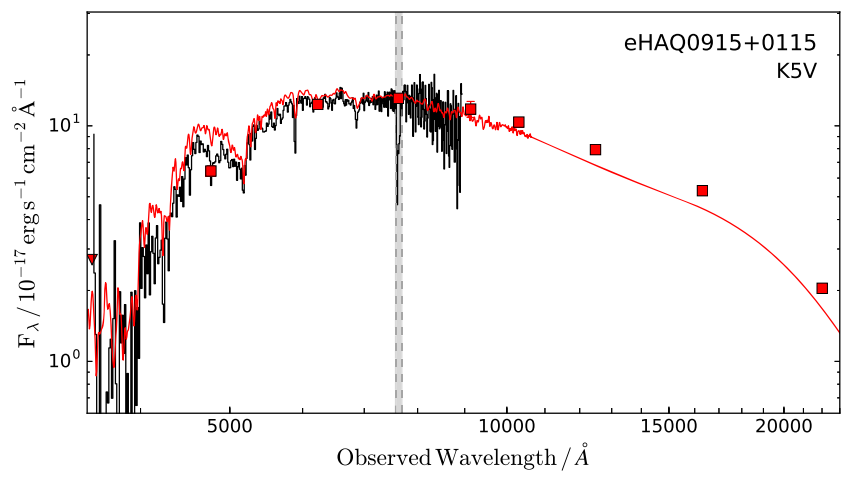

(b)

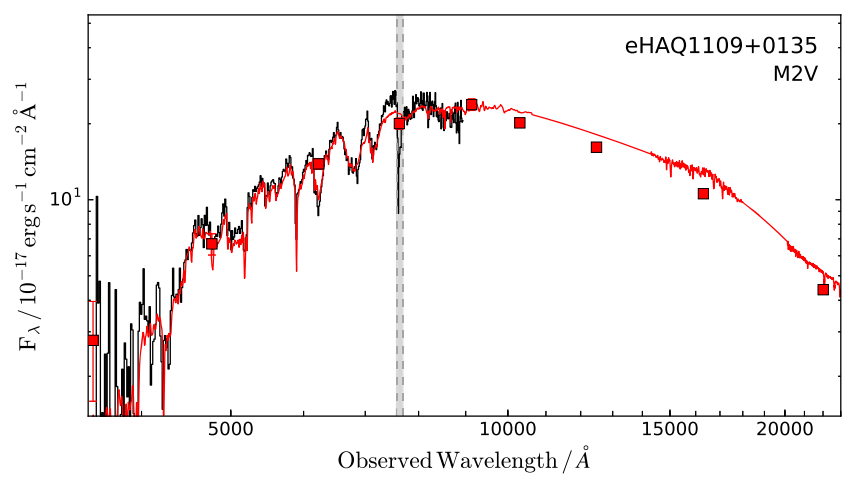

(d)

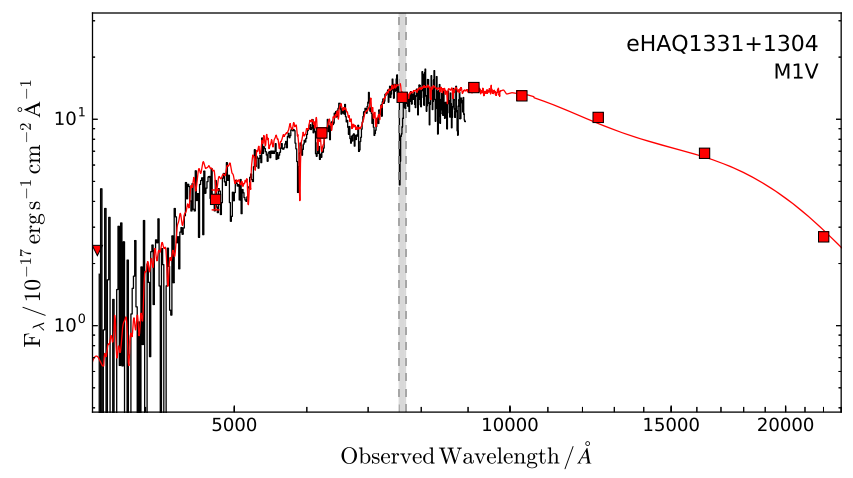

(f)

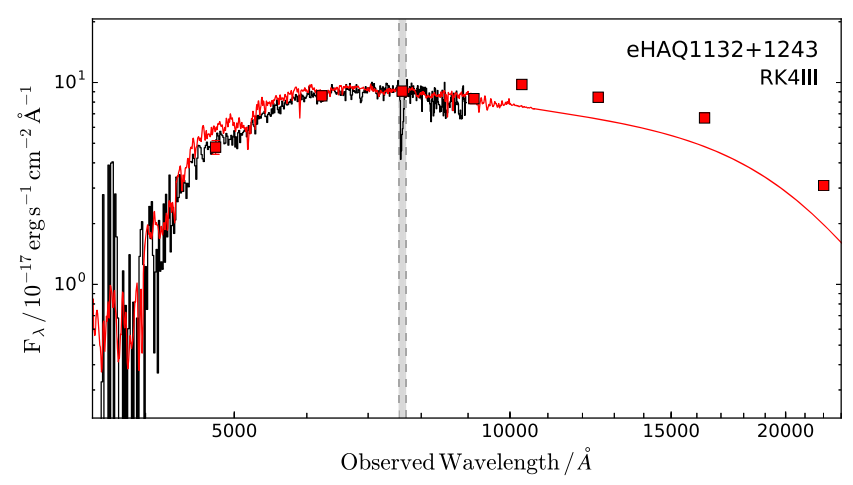

(h)

Figure 7. Spectra of the objects classified as stars. The red line shows the best-fit stellar template. The spectral type is indicated in the upper right corner of each panel. The bottom row (panels (g) and (h)) shows targets with $J-K>-0.05$ mag. 


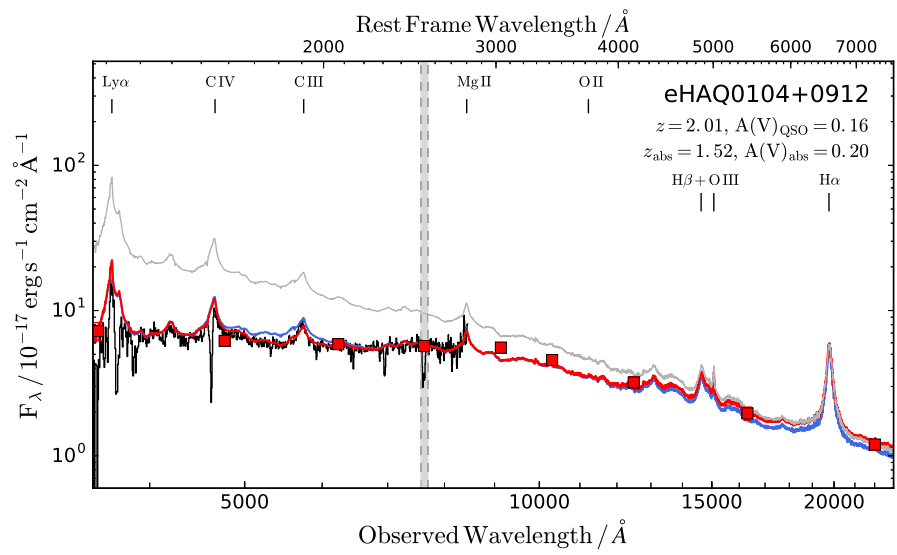

(a)

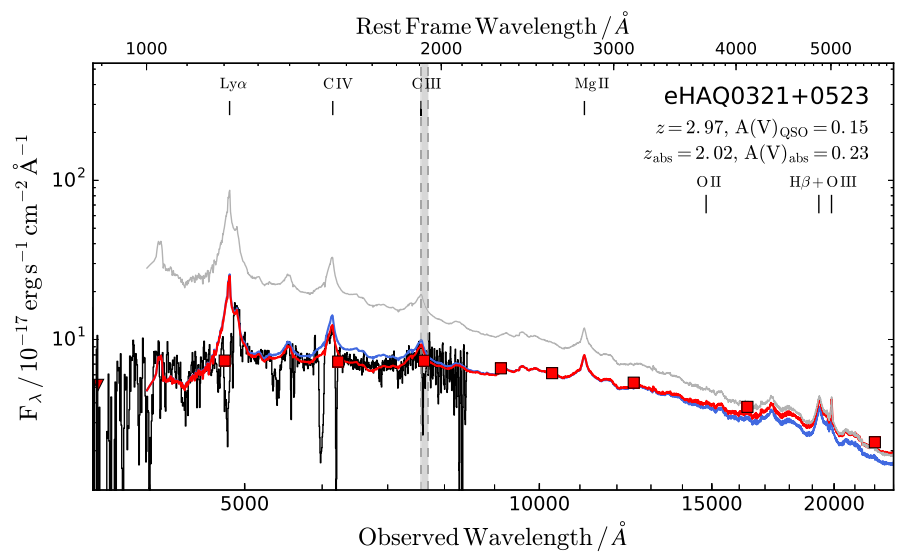

(c)

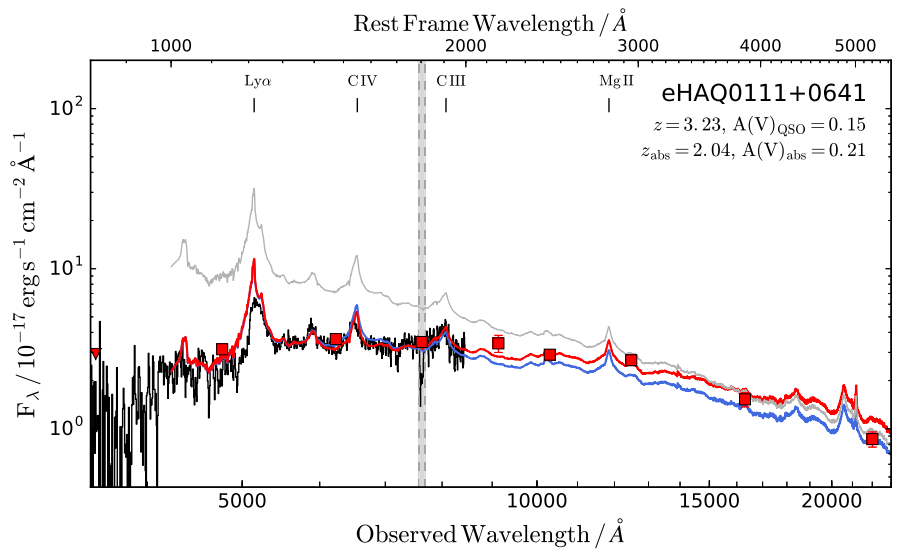

(b)

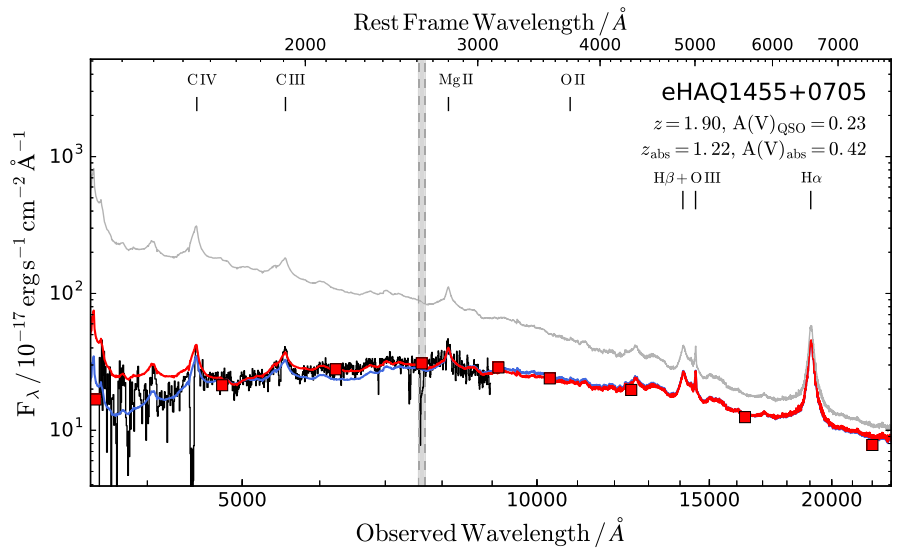

(d)

Figure 8. Statistically identified intervening absorption systems with $2175 \AA$ A dust bump. The upper row (panels (a) and (b)) shows the cases where an absorption-line system has been identified with a spectroscopic redshift consistent with the best-fit $z_{\text {abs. }}$. No absorption-line systems with a corresponding spectroscopic redshift to $z_{\text {abs }}$ have been identified for the cases in the lower row (panels (c) and (d)). The observed spectrum is shown as a solid black curve. The filled squares indicate the SDSS and UKIDSS photometric data points. In the upper right corner we provide the estimated emission redshift, $z$, the amount of (SMC-type) extinction in the quasar's rest-frame, $A(V)_{\mathrm{eso}}$, the best-fit absorption redshift, $z_{\mathrm{abs}}$, and the amount of (LMC-type) extinction in the absorber's rest frame, $A(V)_{\text {abs. }}$ The gray curve shows the quasar template at the estimated redshift of the quasar. The red template shows the best-fit dust model with an intervening dusty absorber at $z_{\text {abs. }}$. For comparison, the blue template indicates the best-fit dust model assuming that all dust is in the quasar. Note that the spectra have not been corrected for telluric absorption (marked with a gray band at $\sim 7600 \AA$ ).

Table 6

Fit Parameters for Intervening Dust Models

\begin{tabular}{lccccrr}
\hline \hline Target & $z_{\text {QSO }}$ & $A(V)_{\text {QSO }}$ & $z_{\text {abs }}$ & $A(V)_{\text {abs }}$ & $L^{\mathrm{a}}$ & $P_{\mathrm{KS}}^{\mathrm{b}}$ \\
\hline eHAQ0104+09 & 2.01 & $0.16 \pm 0.01$ & $1.52 \pm 0.03$ & $0.20 \pm 0.02$ & 92.0 & 0.20 \\
eHAQ0111+06 & 3.23 & $0.15 \pm 0.02$ & $2.04 \pm 0.09$ & $0.21 \pm 0.04$ & 91.1 & 0.12 \\
eHAQ0321+05 & 2.97 & $0.16 \pm 0.01$ & $2.02 \pm 0.02$ & $0.23 \pm 0.02$ & 89.3 & 0.24 \\
eHAQ1455+07 & 1.90 & $0.23 \pm 0.02$ & $1.22 \pm 0.02$ & $0.42 \pm 0.03$ & $1.7 \times 10^{-20}$ \\
\hline
\end{tabular}

Notes. The "null model" refers to the model with only dust in the quasar (i.e., $z_{\mathrm{abs}}=0$ and $A(V)_{\mathrm{abs}}=0$ ). The "general model" refers to the model where both dust in the quasar and dust in the absorber are fitted simultaneously (i.e., with variable $A(V)_{\mathrm{Qso}}, z_{\mathrm{abs}}$, and $\left.A(V)_{\mathrm{abs}}\right)$. For details, see Section 4.1 .1 in Krogager et al. (2015).

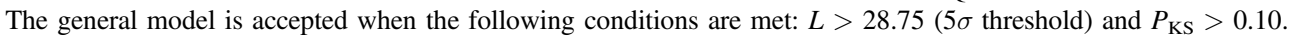

a The logarithm of the likelihood ratio of the maximum likelihood of the null model, $\Lambda_{0}$, and of the general model, $\Lambda_{G} ; L=-2 \ln \left(\Lambda_{0} / \Lambda_{G}\right)$.

${ }^{\mathrm{b}} P$-value from a Kolmogorov-Smirnov test of the normalized residuals from the best-fit general model.

${ }^{c}$ The chance probability of the observed increase in likelihood given the two extra free parameters in the general model.

$$
\text { D.18. eHAQ0147+0411 }(z=1.77)
$$

This is a reddened quasar. Due to possible issues with the flux calibration of the INT spectrum, we have only estimated reddening from the photometry. The object has a detection by NASA's Galaxy Evolution Explorer (GALEX) in two bands (near-UV and far-UV): NUV $=20.59 \pm 0.10 \mathrm{AB}$ mag and $\mathrm{FUV}=20.73 \pm 0.18 \mathrm{AB}$ mag.

\section{D.19. eHAQ0147+1549 (BL Lacertae Object)}

There are no spectral features that can hint at a redshift. The object is detected in radio by NVSS at $1.4 \mathrm{GHz}, F_{1.4 \mathrm{GHz}}=17$ mJy. Given the optical flux, $i=18.3 \mathrm{mag}$, this object is classified as radio loud (following Ivezić et al. 2002). The object exhibits variability between the different epochs of observation. The photometry from SDSS and UKIDSS seems 
to be offset with respect to one another, and the spectrum does not seem to match the SDSS photometry in the $u$ and $g$ bands. These pieces of evidence hint at this object being a BL Lac object. Furthermore, this object appears in the "Catalog of Candidate $\gamma$-ray Blazars" of D'Abrusco et al. (2014).

\section{D.20. $\mathrm{eHAQ0157+1321(z=1.73)}$}

This is a reddened quasar. The spectral shape might be influenced by flux calibration issues for the INT spectra. This object also appears in the KX-catalog of Maddox et al. (2012); however, these authors assign a redshift of $z=0.8524$. This redshift is inconsistent with the data presented here, since we see no evidence of the $\mathrm{H} \beta$ and $\mathrm{O}$ III emission lines, which should be observable at the red end of our spectrum at this redshift. Moreover, our template fitting using only the photometry (excluding the $W_{3}$ and $W_{4}$ bands) yields $z_{\text {phot }}=1.75 \pm 0.10$.

$$
\text { D.21. eHAQ0216+0426 }(z=1.58)
$$

This is a reddened quasar with associated narrow absorption at $z_{\text {abs }}=1.550$.

$$
\text { D.22. eHAQ0227+0521 }(z=2.68)
$$

This is a reddened BAL quasar.

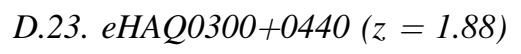

This is a reddened quasar with narrow associated absorption. The spectrum and photometry require a steeper extinction curve than the SMC-like curve from Gordon et al. (2003). We therefore use the extinction curve derived for similar quasars from Zafar et al. (2015) to obtain a better fit.

$$
\text { D.24. eHAQ0321+0523 }(z=2.97)
$$

This is a reddened BAL quasar. The quasar has also been observed in SDSS from which a consistent redshift $\left(z_{\text {SDSS }}=2.9811\right)$ is derived. The quasar also appears in the BALQSO catalog of Allen et al. (2011) and in the catalog of unusual quasars from Meusinger et al. (2012).

$$
\text { D.25. eHAQ0347+0348 }(z=1.85)
$$

This is a reddened quasar.

$$
\text { D.26. eHAQ0828+0313 }(z=1.99)
$$

This is a weak-line quasar with weak BAL features blueward of C IV. We are not able to match the spectrum and photometry simultaneously. However, we note that the template is not suitable for weak-line quasars, since it was derived for "regular" quasars. The NIR photometry is well matched by a quasar template with no dust reddening applied. The quasar appears in the photometric catalogs of Richards et al. (2004, 2009), who estimate photometric redshifts of $z_{\text {phot }}=0.275$ and $z_{\text {phot }}=0.225$, respectively.

$$
\text { D.27. eHAQ0835+0127 }(z=3.30)
$$

This is a reddened quasar with a tentative detection of an Fe II absorption system at $z_{\mathrm{abs}}=1.717$, for which the $\mathrm{Mg}$ II line falls directly on top of the telluric A band.

\section{D.28. eHAQ0835+0830 (Star)}

This is a star of spectral type K4 V.

$$
\text { D.29. } \mathrm{eHAQ0839+0556(z=1.49)}
$$

There are no strong emission lines for this object; however, we observe small absorption features that match up with C IV, $\mathrm{C}$ III], and $\mathrm{Mg}$ II. This redshift is furthermore consistent with the photometric redshift of $z_{\text {phot }}=1.5 \pm 0.1$. The overall SED is well fitted by a continuum template with an intrinsically steep slope with no emission lines and reddened by a steep extinction curve. See Section 4.1.3 for details about the fit.

$$
\text { D.30. } e H A Q 0852+0204(z=2.20)
$$

This is a reddened BAL quasar. The object appears in the catalog of photometric quasar candidates from Richards et al. (2009), who report a photometric redshift of $z_{\text {phot }}=4.255$.

$$
\text { D.31. eHAQ0856+0643 }(z=2.49)
$$

This is a "peculiar" quasar with narrow associated absorption. The optical spectrum is well described by dust reddening $A(V)=0.44$; however, the infrared photometry requires no reddening. The steeper extinction curve of Zafar et al. (2015) does not provide a satisfactory solution either.

$$
\text { D.32. eHAQ0913+0910 }(z=1.95)
$$

This is an FeLoBAL quasar with no apparent reddening due to dust. The large number of blended BAL features in the observed optical spectrum makes it impossible to identify the quasar continuum. This leads to a strong evident mismatch between the optical data and the template. The object appears in the catalog of photometric quasar candidates from Richards et al. (2009), who report a photometric redshift of $z_{\text {phot }}=0.225$.

$$
\text { D.33. eHAQ0915+0115 (Star) }
$$

This is a star of spectral type K5 V.

$$
\text { D.34. eHAQ0919+0843 }(z=2.43)
$$

This is a reddened BAL quasar. The redshift is determined from the $\mathrm{C}$ III] emission line.

$$
\text { D.35. eHAQ0921+0149 }(z=0.99)
$$

This is a highly reddened quasar with narrow emission lines.

$$
\text { D.36. } e H A Q 0923+0520(z=3.40)
$$

This is a reddened BAL quasar. The reddening is determined from fitting the template to the NIR photometry since the intrinsic continuum level is hard to estimate.

$$
\text { D.37. eHAQ0927-0233 }(z=1.24)
$$

This is a reddened quasar possibly with BAL features blueward of $\mathrm{C}$ III]. There is a small deficit in the $Y$ and $J$ bands relative to the best-fit template. This is possibly due to variations in the $\mathrm{Fe}$ II emission around $\mathrm{H} \beta$. The object appears in the catalog of photometric quasar candidates from Richards et al. (2009), who report a photometric redshift of $z_{\text {phot }}=3.875$. 


$$
\text { D.38. eHAQ0930+0148 }(z=2.95)
$$

This is a reddened quasar with narrow associated absorption. We see tentative evidence for a DLA at $z_{\mathrm{DLA}}=2.720$, which matches with metal absorption lines.

$$
\text { D.39. eHAQ0940+0532 }(z=2.32)
$$

This is a reddened quasar with very weak emission lines and high-ionization BALs.

$$
\text { D.40. eHAQ0943+0954 }(z=4.25)
$$

This is a BAL quasar with no evidence for reddening due to dust. The quasar is observed in SDSS with a spectroscopic redshift of $z_{\text {SDSS }}=4.27$ and appears in the catalog of BAL quasars (Allen et al. 2011) and unusual quasars (Meusinger et al. 2012).

$$
\text { D.41. } e H A Q 0943+1300(z=2.68)
$$

This is a highly absorbed BAL quasar with mild reddening and very weak emission lines. The absorption exhibits various velocity profiles, which makes an exact redshift determination difficult. The estimate of reddening is insecure and only based on the NIR photometry.

$$
\text { D.42. eHAQ0949+1207 }(z=3.36)
$$

This is a BAL quasar consistent with no dust reddening.

$$
\text { D.43. eHAQ0950+0440 }(z=2.30)
$$

This is a "peculiar" BAL quasar. The optical spectrum is well described by the quasar template reddened by $A$ $(V)=0.53$; however, the NIR photometry matches neither the reddened nor the unreddened template.

$$
\text { D.44. eHAQ0952+0835 }(z=1.86)
$$

This is a reddened BAL quasar.

$$
\text { D.45. eHAQ1002+0406 (Star) }
$$

This is a star of spectral type M2 V.

$$
\text { D.46. eHAQ1005+0602 }(z=2.57)
$$

This is a reddened BAL quasar.

$$
\text { D.47. eHAQ1010+1158 }(z=2.24)
$$

This is a "peculiar" BAL quasar. The optical spectrum is well described by the dust-reddened quasar template; however, the NIR photometry is consistent with no dust reddening.

$$
\text { D.48. eHAQ1025+1324 }(z=2.57)
$$

This is a "peculiar" BAL quasar. The spectrum is well described by a high amount of reddening $(A(V)=0.78)$, but the NIR photometry requires less reddening $(A(V)=0.15)$. We note that the template may not provide a good description for the optical spectrum, due to the large amount of absorption.

$$
\text { D.49. eHAQ1026-0241 }(z=3.23)
$$

This is a reddened quasar with associated absorption (strongest in $\mathrm{C} \mathrm{IV}, \mathrm{N} \mathrm{V}$, and $\operatorname{Ly} \alpha$ ).

$$
\text { D.50. eHAQ1030+1040 }(z=1.58)
$$

From photometric modeling (using bands from $g$ to $W_{2}$ ), we infer a redshift of $z_{\text {phot }}=4.2 \pm 0.1$, consistent with the estimate from Richards et al. (2009): $z_{\text {phot }}=4.215$. However, the spectrum reveals that this object is indeed an FeLoBAL quasar at lower redshift. The NIR photometry is consistent with no reddening, whereas the optical spectrum seems to require some reddening; however, the estimated $A(V)$ is highly uncertain due to the strong absorption of the continuum.

$$
\text { D.51. eHAQ1106+0844 }(z=1.86)
$$

This is a reddened BAL quasar. The object is also observed by SDSS with a consistent redshift estimate, $z_{\text {SDSS }}=1.8645$, and appears in the catalog of unusual quasars by Meusinger et al. (2012).

\section{D.52. eHAQ1109+0135 (Star)}

This is a star of spectral type M2 V.

$$
\text { D.53. } \mathrm{eHAQ1109+1058(z=1.90)}
$$

This is a reddened quasar with narrow associated absorption. We identify an Mg II absorption system at $z_{\text {abs }}=1.667$. Richards et al. (2009) estimate a photometric redshift of $z_{\text {phot }}=2.735$.

\section{D.54. eHAQ1111+0151 (Star)}

This is a star of spectral type K7 V.

$$
\text { D.55. eHAQ1119+1430 }(z=1.88)
$$

This is a reddened FeLoBAL quasar with weak emission lines.

\section{D.56. eHAQ1120+0812 (Star)}

This is a star of spectral type K5 III.

\section{D.57. eHAQ1132+1243 (Star?)}

The classification of this object is insecure. The best-fit template yields a spectral type of a metal-rich K4 giant. There is an apparent offset between the SDSS and UKIDSS photometry.

$$
\text { D.58. eHAQ1136+0027 }(z=1.12)
$$

This is a highly reddened quasar with associated absorption. The NIR photometry is offset relative to the optical data. This is possibly caused by intrinsic variability in the quasar. The object appears in the photometric catalogs of Richards et al. (2004, 2009), who assign photometric redshifts of $z_{\text {phot }}=0.33$ and $z_{\text {phot }}=3.89$, respectively. The spectroscopic redshift is measured accurately from [O II] $\lambda 3727$.

$$
\text { D.59. eHAQ1144+0902 }(z=1.78)
$$

This is a reddened BAL quasar. The object is also observed by SDSS at a consistent redshift $\left(z_{\mathrm{SDSS}}=1.7849\right)$. The object appears in the BAL catalog of Allen et al. (2011). 


$$
\text { D.60. eHAQ1202+0423 }(z=2.32)
$$

This is a highly absorbed BAL quasar, but we observe no signs of dust reddening.

$$
\text { D.61. eHAQ1203+0652 }(z=2.40)
$$

This is a reddened BAL quasar with weak emission lines.

$$
\text { D.62. eHAQ1203+1118 }(z=3.62)
$$

This is a reddened BAL quasar.

$$
\text { D.63. eHAQ1210+1429 }(z=1.48)
$$

This is a highly reddened quasar with weak emission lines and very strong associated absorption at the quasar redshift $z_{\mathrm{abs}}=1.486$. The optical spectrum is well fitted by a high amount of dust $(A(V)=1.1)$; however, the NIR photometry requires less reddening $(A(V)=0.36$; shown as the blue template in the figure). This indicates that the intrinsic powerlaw slope of the quasar is steeper than average or that the extinction curve is steeper than SMC.

$$
\text { D.64. eHAQ1222+0826 }(z=2.90)
$$

This is a reddened BAL quasar. The NIR photometry is slightly offset compared to the optical data. This is possibly due to intrinsic variability.

$$
\text { D.65. eHAQ1226-0236 }(z=1.25)
$$

This is a reddened ordinary quasar.

$$
\text { D.66. eHAQ1237+1233 }(z=2.31)
$$

This is a reddened BAL quasar with weak emission lines. The optical spectrum is better fitted by a rather high $A(V)$, whereas a fit to the NIR photometry results in a significantly lower amount of reddening: $A(V)=0.12$. We caution the reader that the template does not provide a reasonable description of the optical data, due to the strong absorption. We therefore only provide the $A(V)$ derived from the NIR photometry.

$$
\text { D.67. eHAQ1244+0841 }(z=1.87)
$$

This is a reddened quasar with weak BAL features. We note that the optical spectrum seems to indicate a high $A(V)$ of $0.45 \mathrm{mag}$, but this is highly uncertain due to the possible absorption around C IV and C III]. The object appears in the photometric catalog of Richards et al. (2009), who infer a photometric redshift of $z_{\text {phot }}=2.72$.

$$
\text { D.68. eHAQ1252+0842 }(z=3.53)
$$

This is a BAL quasar. The overall SED is consistent with a low amount of reddening, which could plausibly be ascribed to variations in the intrinsic quasar slope.

$$
\text { D.69. eHAQ1312+1431 }(z=1.64)
$$

This is a reddened quasar with weak C IV and C III] lines. There appears to be absorption from Fe II lines. There is narrow absorption on top of CIV. The object appears in the photometric catalog of Richards et al. (2009), who infer a photometric redshift of $z_{\text {phot }}=3.54$.

$$
\text { D.70. } e H A Q 1326+1317(z=2.00)
$$

This is a strongly absorbed BAL quasar. We see no signs of dust reddening. The object is observed by SDSS $\left(z_{\text {SDSS }}=1.9869\right)$ and is included in the BAL catalog of Allen et al. (2011).

\section{D.71. eHAQ1331+1304 (Star)}

This is a star of spectral type M1 V.

$$
\text { D.72. eHAQ1340+0151 }(z=1.94 \text { ?) }
$$

There are no strong spectral features to securely identify this target. There is a sharp break in the SED after the $z$ band. We tentatively identify the emission feature in the blue edge of the spectrum as $\operatorname{Ly} \alpha$ at redshift $z=1.94$, which matches with the weak absorption feature in the $g$ band. The reddening required for this redshift solution is quite high $(A(V) \sim 1 \mathrm{mag})$ but does not match the NIR photometry. At the same redshift, the NIR photometry requires no reddening. There is no radio detection to aid in classification. The target is marked as a quasar candidate for the Large sky Area Multi-Object Fiber Spectroscopic Telescope (LAMOST) survey (Luo et al. 2015); however, the spectrum observed in LAMOST DR1 shows no flux. The overall SED is fitted well by the continuum model of Richards et al. (2006) with a variable slope and extinction curve. For details, see Section 4.1.3.

$$
\text { D.73. eHAQ1340+1458 }(z=1.73)
$$

This is a reddened quasar with weak BALs in C IV and Si IV. There are possibly variations in the iron pseudo-continuum around $\mathrm{C} \mathrm{III}]$. The NIR data require less reddening ( $A$ $(V) \sim 0.3)$ than the optical data. This indicates that the extinction curve is steeper than SMC or that the intrinsic slope of the quasar power law is steeper than average.

$$
\text { D.74. eHAQ1346+0114 }(z=1.73)
$$

This is a highly reddened quasar with weak C IV emission and blueshifted absorption in both $\mathrm{C}$ IV and $\mathrm{C}$ III]. There are possibly variations in the iron pseudo-continuum around $\mathrm{C}$ III]. The NIR data require less reddening $(A(V) \sim 0.3)$ than the optical data. This indicates that the extinction curve is steeper than SMC or that the intrinsic slope of the quasar power law is steeper than average.

$$
\text { D.75. eHAQ1357-0051 }(z=1.80)
$$

This is a reddened BAL quasar with strong associated lowionization metal absorption $\left(z_{\mathrm{abs}}=1.792\right)$.

$$
\text { D.76. } e H A Q 1400+0720(z=2.40)
$$

This is a reddened BAL quasar with associated lowionization metal absorption at the redshift of the quasar, and possibly also weak Fe II BAL features.

$$
\text { D.77. eHAQ1447+0521 }(z=2.35)
$$

This is a reddened BAL quasar. The object appears in the photometric catalog of Richards et al. (2009), who infer a photometric redshift of $z_{\text {phot }}=4.26$. 


$$
\text { D.78. eHAQ1450+1002 }(z=1.58)
$$

This is a reddened FeLoBAL quasar.

$$
\text { D.79. eHAQ1455+0705 }(z=1.90)
$$

This is a reddened quasar with associated absorption in C IV and $\mathrm{C}$ III]. We see tentative evidence of an intervening $\mathrm{Mg}$ II absorption system at $z_{\mathrm{abs}}=1.650$. Richards et al. (2009) infer a photometric redshift of $z_{\text {phot }}=4.26$. The object is observed by Maddox et al. (2012), who also classify the object as a quasar at redshift $z=1.9094$.

\section{D.80. eHAQ1514-0002 $(z=1.70)$}

This is an FeLoBAL quasar with no evidence of dust reddening from the NIR photometry.

$$
\text { D.81. eHAQ1525+0155 }(z=1.62)
$$

This is a highly reddened quasar with broad absorption around the $\mathrm{C}$ III] emission line, possibly caused by absorption from Fe III, and narrow associated absorption on top of Mg II. We observe blueshifted C IV absorption.

$$
\text { D.82. eHAQ1528+0546 }(z=2.12)
$$

This is a reddened quasar with broad absorption around the $\mathrm{C}$ IV emission line. The object is furthermore observed by SDSS $\left(z_{\text {SDSS }}=2.1051\right)$ and by Maddox et al. (2012), who infer a consistent redshift of $z=2.11$. Moreover, Richards et al. (2009) infer a photometric redshift of $z_{\text {phot }}=0.41$.

$$
\text { D.83. } e H A Q 1539+0351(z=1.63)
$$

This is a reddened BAL quasar. The higher-ionization lines (C IV and Si IV) exhibit stronger BAL features, whereas C III] and $\mathrm{Mg}$ II show progressively weaker lines.

$$
\text { D.84. eHAQ1543+0447 }(z=2.05)
$$

This is a highly reddened quasar with strong excess emission from Fe II and Fe III.

$$
\text { D.85. eHAQ1549+0501 }(z=1.46)
$$

This is a highly reddened quasar with weak emission lines and narrow associated absorption. Richards et al. (2009) infer a photometric redshift of $z_{\text {phot }}=3.88$.

$$
\text { D.86. eHAQ2209+0304 ( } z=2.33)
$$

This is a very "peculiar" quasar. The spectrum reveals very weak emission lines and narrow associated absorption; however, the continuum shape is not matched by the template at all either in the optical or in the NIR.

$$
\text { D.87. eHAQ2235+0635 }(z=1.13)
$$

This is a highly reddened quasar with associated lowionization metal absorption. The object is detected in the FIRST survey at $1.4 \mathrm{GHz}, F_{1.4 \mathrm{GHz}}=0.62 \pm 0.14 \mathrm{mJy}$.

$$
\text { D.88. eHAQ2236+0731 }(z=1.72)
$$

This is a reddened quasar categorized as an optical variable in the Palomar-QUEST survey (Bauer et al. 2009).

$$
\text { D.89. } e H A Q 2247+0922(z=1.63)
$$

This is a reddened quasar.

$$
\text { D.90. } e H A Q 2255+1213(z=2.05)
$$

This is a reddened quasar with broad absorption around C IV and $\mathrm{C}$ III] possibly caused by a combination of weak highionization BAL and FeLoBAL features. The iron absorption would also explain the deficit observed in the $Y$ and $J$ bands. The object is detected in the FIRST survey at $1.4 \mathrm{GHz}$, $F_{1.4 \mathrm{GHz}}=4.19 \pm 0.16 \mathrm{mJy}$.

$$
\text { D.91. eHAQ2256+0531 }(z=1.35)
$$

This is a highly reddened quasar with weak emission lines and narrow associated absorption. We observe a large excess in the $H$ and $K$ bands, most likely due to the $\mathrm{H} \alpha$ line in the $H$ band and emission from the host galaxy and hot dust that start to become important in the $K$ band (rest frame $\sim 1 \mu \mathrm{m}$ ). The object is a known radio source detected at several frequencies. It is detected in FIRST and NVSS at $1.4 \mathrm{GHz}$ with a flux of $F_{\text {FIRST }}=11.99 \pm 0.13$ and $F_{\mathrm{NVSS}}=14.8 \pm 0.6 \mathrm{mJy}$, respectively. Jackson et al. (2007) report a flux at $8.4 \mathrm{GHz}$ of $F_{8.4 \mathrm{GHz}}=10.7 \pm 0.2 \mathrm{mJy}$, and Coble et al. (2007) report a flux at $28.5 \mathrm{GHz}$ of $F_{28.5 \mathrm{GHz}}=8.15 \pm 0.15 \mathrm{mJy}$.

$$
\text { D.92. eHAQ2258+0251 }(z=2.43)
$$

This is a reddened BAL quasar.

$$
\text { D.93. eHAQ2259+0208 }(z=1.83)
$$

This is a reddened quasar.

$$
\text { D.94. } e H A Q 2259+0256(z=1.83)
$$

This is a reddened quasar with broad absorption around the $\mathrm{C}$ IV and C III] emission lines. The object is detected in the FIRST survey at $1.4 \mathrm{GHz}, F_{1.4 \mathrm{GHz}}=0.72 \pm 0.13 \mathrm{mJy}$.

$$
\text { D.95. eHAQ2259+0736 }(z=0.78)
$$

This is a highly reddened quasar with narrow emission lines and associated narrow absorption. We can accurately measure the redshift from $[\mathrm{O}$ II] $\lambda 3727$.

$$
\text { D.96. } e H A Q 2301+0752(z=2.62)
$$

This is a reddened BAL quasar.

$$
\text { D.97. eHAQ2303+0747 }(z=1.31)
$$

The spectrum from INT does not match the photometry due to issues in the flux calibration. We therefore only use the spectrum to securely classify this object as a quasar on the basis of the broad emission line ( $\mathrm{Mg}$ II at $z=1.31$ ). We estimate the reddening using only the photometry. The object is detected in the two bands of GALEX (near-UV and far-UV): $\mathrm{NUV}=22.7 \pm 0.2 \mathrm{AB}$ mag and FUV $>23.2 \mathrm{AB}$ mag.

$$
\text { D.98. eHAQ2309+1159 }(z=2.00)
$$

This is a reddened quasar with absorption in the red wing of $\mathrm{C}$ III] possibly due to Fe III absorption. 


$$
\text { D.99. } e H A Q 2310+0447(z=1.42)
$$

This is a reddened BAL quasar.

$$
\text { D.100. } e H A Q 2313+1259(z=2.11)
$$

This is a reddened quasar with narrow associated absorption on top of the C IV emission line. The reddening estimate is uncertain due to noise in the blue edge of the spectrum.

$$
\text { D.101. eHAQ2314+0552 }(z=2.03)
$$

This is a reddened quasar with narrow emission lines and broad emission around the CIV and C III] emission lines possibly due to a blend of weak high-ionization BALs and lowionization iron absorption.

$$
\text { D.102. } e H A Q 2316+0651(z=1.37)
$$

This is a reddened quasar with associated absorption at the quasar redshift. We observe strong absorption blueward of $\mathrm{C}$ III], possibly due to BAL features.

$$
\text { D.103. eHAQ2321+1107 }(z=1.56)
$$

This is a reddened BAL quasar.

$$
\text { D.104. eHAQ2321+1121 }(z=2.18)
$$

This is a reddened quasar. We observe a deficit in the $J$ and $H$ bands compared to the best-fit template. This can plausibly be explained by variations in the iron pseudo-continuum. This would also explain the small excess in the spectrum around the $z$ band.

$$
\text { D.105. } e H A Q 2334+1519(z=2.23)
$$

This is a reddened BAL quasar with weak absorption from low-ionization iron lines. The NIR photometry is offset from the optical data possibly due to intrinsic variability.

$$
\text { D. 106. } e H A Q 2344+1416(z=2.52)
$$

This is a reddened BAL quasar possibly with variations in the iron pseudo-continuum around C IV and C IIII. The object is detected in the FIRST survey at $1.4 \mathrm{GHz}, F_{1.4 \mathrm{GHz}}=0.97 \pm$ $0.16 \mathrm{mJy}$.

$$
\text { D.107. } e H A Q 2358+1030(z=2.05)
$$

This is a highly reddened quasar with very strong and narrow emission lines. We clearly detect the He II emission line redward of C IV. We furthermore observe an emission line at $\lambda_{\text {obs }}=6988.8 \AA$, which is caused by second-order contamination from Ly $\alpha$. This quasar is described in detail in Heintz et al. (2016).

$$
\text { D.108. eHAQ2359+1354 ( } z=2.79)
$$

We observed this target with grism \#6 to look for a DLA around $z_{\text {abs }}=2.248$. We see evidence for metal absorption from Fe II and Si II and a sharp drop around $4000 \AA$, which corresponds to the location of the Ly $\alpha$ line from the DLA. We only use the spectrum to fix the redshift and subsequently determine the reddening by fitting the BOSS spectrum.

\section{REFERENCES}

Allen, J. T., Hewett, P. C., Maddox, N., Richards, G. T., \& Belokurov, V. 2011, MNRAS, 410, 860

Baloković, M., Smolčić, V., Ivezić, Ž., et al. 2012, ApJ, 759, 30

Banerji, M., McMahon, R. G., Hewett, P. C., et al. 2012, MNRAS, 427, 2275

Banerji, M., McMahon, R. G., Hewett, P. C., Gonzalez-Solares, E., \& Koposov, S. E. 2013, MNRAS, 429, L55

Bauer, A., Baltay, C., Coppi, P., et al. 2009, ApJ, 705, 46

Benn, C. R., Vigotti, M., Carballo, R., Gonzalez-Serrano, J. I., \& Sánchez, S. F. 1998, MNRAS, 295, 451

Butler, N. R., \& Bloom, J. S. 2011, AJ, 141, 93

Coble, K., Bonamente, M., Carlstrom, J. E., et al. 2007, AJ, 134, 897

Condon, J. J., Cotton, W. D., Greisen, E. W., et al. 1998, AJ, 115, 1693

Cooke, R. J., Pettini, M., \& Jorgenson, R. A. 2015, ApJ, 800, 12

Croom, S. M., Smith, R. J., Boyle, B. J., et al. 2004, MNRAS, 349, 1397

D’Abrusco, R., Massaro, F., Paggi, A., et al. 2014, ApJS, 215, 14

Dawson, K. S., Schlegel, D. J., Ahn, C. P., et al. 2013, AJ, 145, 10

De Cia, A., Ledoux, C., Savaglio, S., Schady, P., \& Vreeswijk, P. M. 2013, A\&A, 560, A88

Draine, B. T. 2003, ARA\&A, 41, 241

Draine, B. T., \& Lee, H. M. 1984, ApJ, 285, 89

Ellison, S. L., Hall, P. B., \& Lira, P. 2005, AJ, 130, 1345

Ellison, S. L., Pettini, M., Steidel, C. C., \& Shapley, A. E. 2001, ApJ, 549, 770

Ellison, S. L., York, B. A., Pettini, M., \& Kanekar, N. 2008, MNRAS, 388, 1349

Fall, S. M., \& Pei, Y. C. 1989, ApJ, 337, 7

Fall, S. M., Pei, Y. C., \& McMahon, R. G. 1989, ApJL, 341, L5

Fleuren, S., Sutherland, W., Dunne, L., et al. 2012, MNRAS, 423, 2407

Foreman-Mackey, D., Hogg, D. W., Lang, D., \& Goodman, J. 2013, PASP, 125,306

Frank, S., \& Péroux, C. 2010, MNRAS, 406, 2235

Fynbo, J. P. U., Krogager, J.-K., Venemans, B., et al. 2013, ApJS, 204, 6

Fynbo, J. P. U., Ledoux, C., Noterdaeme, P., et al. 2011, MNRAS, 413, 2481

Glikman, E., Helfand, D. J., \& White, R. L. 2006, ApJ, 640, 579

Glikman, E., Helfand, D. J., White, R. L., et al. 2007, ApJ, 667, 673

Glikman, E., Urrutia, T., Lacy, M., et al. 2012, ApJ, 757, 51

Glikman, E., Urrutia, T., Lacy, M., et al. 2013, ApJ, 778, 127

Goobar, A. 2008, ApJL, 686, L103

Gordon, K. D., Clayton, G. C., Misselt, K. A., Landolt, A. U., \& Wolff, M. J. 2003, ApJ, 594, 279

Graham, M. J., Djorgovski, S. G., Drake, A. J., et al. 2014, MNRAS, 439, 703

Gregg, M. D., Lacy, M., White, R. L., et al. 2002, ApJ, 564, 133

Hainline, K. N., Hickox, R. C., Carroll, C. M., et al. 2014, ApJ, 795, 124

Hall, P. B., Anderson, S. F., Strauss, M. A., et al. 2002, ApJS, 141, 267

Heintz, K. E., Fynbo, J. P. U., \& Høg, E. 2015, A\&A, 578, A91

Heintz, K. E., Fynbo, J. P. U., Krogager, J.-K., et al. 2016, AJ, 152, 13

Hopkins, P. F., Hernquist, L., Martini, P., et al. 2005, ApJL, 625, L71

Hopkins, P. F., Somerville, R. S., Hernquist, L., et al. 2006, ApJ, 652, 864

Hopkins, P. F., Strauss, M. A., Hall, P. B., et al. 2004, AJ, 128, 1112

Howk, J. C., Wolfe, A. M., \& Prochaska, J. X. 2005, ApJL, 622, L81

Ivezić, Ž., Menou, K., Knapp, G. R., et al. 2002, AJ, 124, 2364

Jackson, N., Battye, R. A., Browne, I. W. A., et al. 2007, MNRAS, 376, 371

Jenkins, E. B., \& Peimbert, A. 1997, ApJ, 477, 265

Jiang, P., Ge, J., Prochaska, J. X., et al. 2010, ApJ, 724, 1325

Jorgenson, R. A., Wolfe, A. M., Prochaska, J. X., et al. 2006, ApJ, 646, 730

Jorgenson, R. A., Wolfe, A. M., \& Prochaska, J. X. 2010, ApJ, 722, 460

Kanekar, N., Prochaska, J. X., Smette, A., et al. 2014, MNRAS, 438, 2131

Kaplan, K. F., Prochaska, J. X., Herbert-Fort, S., Ellison, S. L., \& Dessauges-Zavadsky, M. 2010, PASP, 122, 619

Khare, P., vanden Berk, D., York, D. G., Lundgren, B., \& Kulkarni, V. P. 2012, MNRAS, 419, 1028

Koo, D. C., Kron, R. G., \& Cudworth, K. M. 1986, PASP, 98, 285

Krawczyk, C. M., Richards, G. T., Gallagher, S. C., et al. 2015, AJ, 149, 203

Krogager, J.-K., Fynbo, J. P. U., Noterdaeme, P., et al. 2016, MNRAS, 455,2698

Krogager, J.-K., Geier, S., Fynbo, J. P. U., et al. 2015, ApJS, 217, 5

Lacy, M., Petric, A. O., Sajina, A., et al. 2007, AJ, 133, 186

Lawrence, A., Warren, S. J., Almaini, O., et al. 2007, MNRAS, 379, 1599

Ledoux, C., Noterdaeme, P., Petitjean, P., \& Srianand, R. 2015, A\&A, 580, A8

Ledoux, C., Petitjean, P., \& Srianand, R. 2003, MNRAS, 346, 209

Leighly, K. M., Cooper, E., Grupe, D., Terndrup, D. M., \& Komossa, S. 2015, ApJL, 809, L13

Leighly, K. M., Terndrup, D. M., Baron, E., et al. 2014, ApJ, 788, 123

Luo, A.-L., Zhao, Y.-H., Zhao, G., et al. 2015, RAA, 15, 1095 
Maddox, N., Hewett, P. C., Péroux, C., Nestor, D. B., \& Wisotzki, L. 2012, MNRAS, 424, 2876

Maddox, N., Hewett, P. C., Warren, S. J., \& Croom, S. M. 2008, MNRAS, 386, 1605

Meusinger, H., Schalldach, P., Mirhosseini, A., \& Pertermann, F. 2016, A\&A, 587, A83

Meusinger, H., Schalldach, P., Scholz, R.-D., et al. 2012, A\&A, 541, A77

Murphy, M. T., \& Bernet, M. L. 2016, MNRAS, 455, 1043

Murphy, M. T., \& Liske, J. 2004, MNRAS, 354, L31

Neeleman, M., Prochaska, J. X., \& Wolfe, A. M. 2015, ApJ, 800, 7

Netzer, H. 2015, ARA\&A, 53, 365

Noterdaeme, P., Krogager, J. K., Balashev, S., et al. 2016, in press, arXivpreprint: 1609.01422

Noterdaeme, P., Laursen, P., Petitjean, P., et al. 2012b, A\&A, 547, L1

Noterdaeme, P., Petitjean, P., Carithers, W. C., et al. 2012a, A\&A, 540, A63

Noterdaeme, P., Petitjean, P., Ledoux, C., \& Srianand, R. 2009, A\&A, 505, 1087

Noterdaeme, P., Petitjean, P., \& Srianand, R. 2015a, A\&A, 578, L5

Noterdaeme, P., Petitjean, P., Srianand, R., Ledoux, C., \& Le Petit, F. 2007, A\&A, 469, 425

Noterdaeme, P., Srianand, R., Rahmani, H., et al. 2015b, A\&A, 577, A24

Pei, Y. C., Fall, S. M., \& Bechtold, J. 1991, ApJ, 378, 6

Peters, C. M., Richards, G. T., Myers, A. D., et al. 2015, ApJ, 811, 95

Peth, M. A., Ross, N. P., \& Schneider, D. P. 2011, AJ, 141, 105

Pickles, A. J. 1998, PASP, 110, 863

Planck Collaboration et al. 2014, A\&A, 571, A16

Polletta, M. d. C., Wilkes, B. J., Siana, B., et al. 2006, ApJ, 642, 673

Pontzen, A., \& Pettini, M. 2009, MNRAS, 393, 557

Prochaska, J. X., Herbert-Fort, S., \& Wolfe, A. M. 2005, ApJ, 635, 123

Prochaska, J. X., \& Wolfe, A. M. 2009, ApJ, 696, 1543

Richards, G. T., Fan, X., Newberg, H. J., et al. 2002, AJ, 123, 2945
Richards, G. T., Hall, P. B., Vanden Berk, D. E., et al. 2003, AJ, 126, 1131 Richards, G. T., Lacy, M., Storrie-Lombardi, L. J., et al. 2006, ApJS, 166,470

Richards, G. T., Myers, A. D., Gray, A. G., et al. 2009, ApJS, 180, 67

Richards, G. T., Myers, A. D., Peters, C. M., et al. 2015, ApJS, 219, 39

Richards, G. T., Nichol, R. C., Gray, A. G., et al. 2004, ApJS, 155, 257

Ross, N. P., Myers, A. D., Sheldon, E. S., et al. 2012, ApJS, 199, 3

Sanders, D. B., Soifer, B. T., Elias, J. H., et al. 1988a, ApJ, 325, 74

Sanders, D. B., Soifer, B. T., Elias, J. H., Neugebauer, G., \& Matthews, K. 1988b, ApJL, 328, L35

Schlafly, E. F., \& Finkbeiner, D. P. 2011, ApJ, 737, 103

Schmidt, K. B., Marshall, P. J., Rix, H.-W., et al. 2010, ApJ, 714, 1194

Selsing, J., Fynbo, J. P. U., Christensen, L., \& Krogager, J.-K. 2016, A\&A, 585, A87

Srianand, R., Noterdaeme, P., Ledoux, C., \& Petitjean, P. 2008, A\&A, 482, L39

Srianand, R., Petitjean, P., Ledoux, C., Ferland, G., \& Shaw, G. 2005 MNRAS, 362, 549

Stern, D., Assef, R. J., Benford, D. J., et al. 2012, ApJ, 753, 30

Urrutia, T., Becker, R. H., White, R. L., et al. 2009, ApJ, 698, 1095

van Dokkum, P. G. 2001, PASP, 113, 1420

Vestergaard, M., \& Wilkes, B. J. 2001, ApJS, 134, 1

Vladilo, G., \& Péroux, C. 2005, A\&A, 444, 461

Vladilo, G., Prochaska, J. X., \& Wolfe, A. M. 2008, A\&A, 478, 701

Wang, J.-G., Zhou, H.-Y., Ge, J., et al. 2012, ApJ, 760, 42

Warren, S. J., Hambly, N. C., Dye, S., et al. 2007, MNRAS, 375, 213

Warren, S. J., Hewett, P. C., \& Foltz, C. B. 2000, MNRAS, 312, 827

Wolfe, A. M., Gawiser, E., \& Prochaska, J. X. 2005, ARA\&A, 43, 861

Wright, E. L., Eisenhardt, P. R. M., Mainzer, A. K., et al. 2010, AJ, 140, 1868

York, D. G., Adelman, J., Anderson, Jr., J. E., et al. 2000, AJ, 120, 1579

Zafar, T., Møller, P., Watson, D., et al. 2015, A\&A, 584, A100 